\title{
New Neumerical Method to Calculate Time-Dependent Quantum Properties in Finite Temperature Based on the Heisenberg Equation of Motion
}

\author{
Shin-Ichiro Kondo \\ Department of Materials Science and Engineering, Nagasaki University, Nagasaki, Japan \\ Email:kondou@nagasaki-u.ac.jp
}

Received August 15, 2012; revised September 14, 2012; accepted September 19, 2012

\begin{abstract}
For the purpose of computer calculation to evaluate time-dependent quantum properties in finite temperature, we propose new numerical method expressed in the forms of simultaneous differential equations. At first we derive the equation of motion in finite temperature, which is found to be same expression as Heisenberg equation of motion except for the $c$-number. Based on this equation, we construct numerical method to estimate time-dependent physical properties in finite temperature precisely without using analytical procedures such as Keldysh formalism. Since our approach is so simple and is based on the simultaneous differential equations including no terms related to self-energies, computer programming can be easily performed. It is possible to estimate exact time-dependent physical properties, providing that Hamiltonian of the system is taken to be a one-electron picture. Furthermore, we refer to the application to the many body problem and it is numerically possible to calculate physical properties using Hartree Fock approximation. Our numerical method can be applied to the case even when perturbative Hamiltonians are newly introduced or Hamiltonian shows complex time-dependent behavior. In this article, at first, we derive the equation of motion in finite temperature. Secondly, for the purpose of verification and of exhibiting the usefulness, we show the derivation of gap equation of superconductivity and of sum rule of electrical conductivity and the application to the many body problem. Finally we apply this method to these two cases: the first case is most simplified resonance charge transfer neutralization of an ion and the second is the same process but impurity potential is newly introduced as perturbative Hamiltonian. Through both cases, it is found that neutralization process is not so sensitive to temperature, however, impurity potential as small as $10 \mathrm{meV}$ strongly influences the neutralization of ion.
\end{abstract}

Keywords: Heisenberg Equation of Motion; Neumann Equation; Time-Dependent Physical Properties; Finite Temperature; Numerical Solutions; Simultaneous Differential Equations

\section{Introduction}

In the previous work [1], we proposed a new and numerical method so as to evaluate various time-dependent properties and to extend the theoretical forms easily even when Hamiltonian shows complex time-dependence or perturbative Hamiltonian is newly introduced. Our proposed method is based on the Heisenberg equations of motion as shown below

$$
\mathrm{i} \hbar \frac{\partial}{\partial t} \hat{a}_{i}^{\dagger}(t)=\left[\hat{a}_{i}^{\dagger}(t), \hat{H}\right]
$$

where operator $\hat{a}_{i}^{\dagger}(t)$ is in Heisenberg representation. By using Equation (1.1), the differentiation of

$\hat{a}_{i}^{\dagger}(t) \hat{a}_{j}(t)$ can be expressed as

$$
\begin{aligned}
\frac{\partial}{\partial t} \hat{a}_{i}^{\dagger}(t) \hat{a}_{j}(t)= & \frac{1}{\mathrm{i} \hbar}\left[\hat{a}_{i}^{\dagger}(t), \hat{H}\right] \hat{a}_{j}(t) \\
& +\frac{1}{\mathrm{i} \hbar} \hat{a}_{i}^{\dagger}(t)\left[\hat{a}_{j}(t), \hat{H}\right] .
\end{aligned}
$$

By taking the expectation value of each term, we obtain the following differential equation:

$$
\begin{aligned}
\frac{\mathrm{d}}{\mathrm{d} t}\left\langle\hat{a}_{i}^{\dagger}(t) \hat{a}_{j}(t)\right\rangle= & \frac{1}{\mathrm{i} \hbar}\left\langle\left[\hat{a}_{i}^{\dagger}(t), \hat{H}\right] \hat{a}_{j}(t)\right\rangle \\
& +\frac{1}{\mathrm{i} \hbar}\left\langle\hat{a}_{i}^{\dagger}(t)\left[\hat{a}_{j}(t), \hat{H}\right]\right\rangle
\end{aligned}
$$

However, as shown above, theoretical treatments are restricted to the ground state, i.e., $T=0 \mathrm{~K}$; thus various time-dependent physical properties in finite temperature 
have remained unresolved.

As to the theoretical evaluation of time-dependent properties in finite temperature, linear response theory [2] is an excellent method and can be applied to many cases such as calculations of electrical conductivity and magnetic susceptibility under the oscillating field and it is proved that this method gives exact solutions within the range of linearity. Usually time-dependent perturbative Hamiltonian $\hat{H}_{1}(t)$ is given in the form of separation of variables in the linear response theory:

$$
\hat{H}_{1}(t)=-f(t) \hat{A}
$$

By defining perturbative Hamiltonian in the form of separation of variables as shown in Equation (1.4), various time-dependent physical properties $\langle\hat{B}(t)\rangle_{\mathrm{av}}$ in finite temperature, which originates from the operator $\hat{B}$, can be easily deduced as

$$
\langle\hat{B}(t)\rangle_{\mathrm{av}}=-\frac{1}{\hbar} \int_{-\infty}^{\infty} \mathrm{d} \tau f(\tau) g_{B A}^{R}(t-\tau)
$$

where $g_{B A}^{R}(t)$ is a retarded thermal Green function and defined by

$$
g_{B A}^{R}(t) \equiv-\hbar \theta(t) \phi_{B A}(t)
$$

and

$$
\begin{gathered}
\phi_{B A}(t) \equiv \frac{\mathrm{i}}{\hbar} \operatorname{Tr}\left\{\hat{\rho}_{0}\left[\hat{B}_{H}(t), \hat{A}\right]\right\} \\
\hat{B}_{H}(t) \equiv \exp \left(\mathrm{i} \hat{H}_{0} t / \hbar\right) \hat{B} \exp \left(-\mathrm{i} \hat{H}_{0} t / \hbar\right)
\end{gathered}
$$

As shown in Equations (1.5)-(1.8), if a perturbative Hamiltonian can not be decomposed into the form of separation of variables of Equation (1.4) or can show complex time-dependent behaviours, it seems to be a complicated task to evaluate time-dependent physical properties $\langle\hat{B}(t)\rangle_{\text {av }}$ because of analytical and/or numerical difficulty in the estimation of retarded thermal Green function $g_{B A}^{R}(t)$ and of analytical corrections concerning to Equations (1.5)-(1.8). In addition, linear response theory can not be applied unless linearity is observed.

Although Green function methods are excellent and smart in analyzing the various time dependent phenomena, integral schemes are essential in diagram methods (Dyson equation), which easily leads to numerical and/or analytical difficulties and awkwardness for the explanation of experimental data. For example, let us consider the simplest case of atom-surface collision where the electron transfer matrix between surface and an ion and energy level of the ion shows strong time-dependence due to the change in atom-surface distance, we can write down the time-dependent Newns-Anderson Hamiltonian as below

$$
\begin{aligned}
& \hat{H}(z)=\hat{H}_{0}(z)+\hat{H}_{1}(z) \\
& \hat{H}_{0}(z)=\sum_{k} E_{k} \hat{C}_{k}^{\dagger} \hat{C}_{k}+E_{a}(z) \hat{C}_{a}^{\dagger} \hat{C}_{a}, \\
& \hat{H}_{1}(z)=\sum_{k}\left(V_{a k}(z) \hat{C}_{a}^{\dagger} \hat{C}_{k}+\text { h.c. }\right) .
\end{aligned}
$$

where $z$ is an atom-surface distance with showing timedependence $\left(z=v|t| \quad v\right.$ : velocity of ion); $E_{k}$ is the energy of a conduction electron with momentum $k ; \hat{C}_{k}^{\dagger}$ and $\hat{C}_{k}$ are creation and annihilation operators of a conduction electron with momentum $k ; E_{a}(z)$ denotes the energy level of an ion and usually depends on the $z$; $\hat{C}_{a}^{\dagger}$ and $\hat{C}_{a}$ are creation and annihilation operators of a state of the ion, respectively; and $V_{a k}(z)$ is the electron transfer matrix element from the conduction electron $\boldsymbol{k}$ to a state of the ion and can be expressed as a function of $z$. Keldysh formalism is usually adopted in treating such a non-equilibirum problem and Dyson equation is given in the following form:

$$
\begin{aligned}
& G\left(t, t^{\prime}\right)=G^{0}\left(t, t^{\prime}\right) \\
& +\left(\frac{1}{\hbar}\right)^{2} \int_{-\infty}^{\infty} \int_{-\infty}^{\infty} \mathrm{d} t_{1} \mathrm{~d} t_{2} G^{0}\left(t, t_{1}\right) \Sigma\left(t_{1}, t_{2}\right) G\left(t_{2}, t^{\prime}\right)
\end{aligned}
$$

where $G\left(t, t^{\prime}\right)$ is retaded Green function of ion and is expressed as

$$
\begin{gathered}
G\left(t, t^{\prime}\right)=-\mathrm{i} \theta\left(t-t^{\prime}\right)\left\langle\left\{\hat{C}_{a}(t), \hat{C}_{a}^{\dagger}\left(t^{\prime}\right)\right\}\right\rangle \\
\hat{C}_{a}(t) \equiv \exp \left(\mathrm{i} \int_{0}^{t} H(\tau) \mathrm{d} \tau / \hbar\right) \hat{C}_{a} \exp \left(-\mathrm{i} \int_{0}^{t} H(\tau) \mathrm{d} \tau / \hbar\right)
\end{gathered}
$$

$G^{0}\left(t, t^{\prime}\right)$ and $\Sigma\left(t_{1}, t_{2}\right)$ are retarded Green function of non-perturative state of ion and self-energy, respectively.

$$
\begin{gathered}
G^{0}(t, t)=-\mathrm{i} \theta\left(t-t^{\prime}\right) \exp \left(-\frac{\mathrm{i}}{\hbar} \int_{t^{\prime}}^{t} E_{a}(\tau) \mathrm{d} \tau\right) \\
\Sigma\left(t_{1}, t_{2}\right)=\sum_{\boldsymbol{k}} V_{a \boldsymbol{k}}\left(t_{1}\right) G_{\boldsymbol{k}}^{0}\left(t_{1}, t_{2}\right) V_{\boldsymbol{k} a}\left(t_{2}\right)
\end{gathered}
$$

And

$$
\begin{gathered}
G_{\boldsymbol{k}}^{0}\left(t_{1}, t_{2}\right)=-\mathrm{i} \theta\left(t_{1}-t_{2}\right) \exp \left[-\frac{\mathrm{i}}{\hbar}\left(t_{1}-t_{2}\right) E_{\boldsymbol{k}}\right] \\
\text { Assuming } V_{a \boldsymbol{k}}(t)=V_{\boldsymbol{k}}^{0} \mu(t), \text { then } \Sigma\left(t_{1}, t_{2}\right) \text { is } \\
\begin{aligned}
\Sigma\left(t_{1}, t_{2}\right)= & -\mathrm{i} \theta\left(t_{1}-t_{2}\right) \mu\left(t_{1}\right) \mu\left(t_{2}\right) \frac{1}{\pi} \int_{-\infty}^{\infty} \mathrm{d} x \pi \sum_{\boldsymbol{k}}\left|V_{\boldsymbol{k}}^{0}\right|^{2} \\
& \times \delta\left(x-E_{k}\right) \exp \left(-\mathrm{i}\left(t_{1}-t_{2}\right) x / \hbar\right) \\
= & -\mathrm{i} \theta\left(t_{1}-t_{2}\right) \mu\left(t_{1}\right) \mu\left(t_{2}\right) \\
& \times \frac{1}{\pi} \int_{-\infty}^{\infty} \mathrm{d} x \Delta(x) \exp \left(-\mathrm{i}\left(t_{1}-t_{2}\right) x / \hbar\right)
\end{aligned}
\end{gathered}
$$


where

$$
\Delta(x)=\pi \sum_{k}\left|V_{k}^{0}\right|^{2} \delta\left(x-E_{k}\right)
$$

The number of electron on the ion at $t \rightarrow \infty$, i.e., $n(\infty)$, which we should seek and calculate, is

$$
n(\infty)=n(-\infty)|G(\infty,-\infty)|^{2}+\sum_{k} n_{k}(-\infty)\left|G_{k}(\infty,-\infty)\right|^{2}
$$

In above equation, $n_{k}(-\infty)$ denotes the number of electron occupying the band state $\boldsymbol{k}$ at initial state and $G_{\boldsymbol{k}}\left(t, t^{\prime}\right)$ is given

$$
G_{\boldsymbol{k}}\left(t, t^{\prime}\right)=\frac{V_{\boldsymbol{k}}^{0}}{\hbar} \int_{-\infty}^{\infty} G\left(t, t_{1}\right) \mu\left(t_{1}\right) G_{\boldsymbol{k}}^{0}\left(t_{1}, t^{\prime}\right) \mathrm{d} t_{1}
$$

As shown in the series of Equations (1.12) to (1.21), many calculations and procedures are necessary to evaluate $n(\infty)$, which suggest that analytical efforts to evaluate $n(\infty)$ are usually failed except for a few cases. Since energy state of ion $E_{a}(t)$, which is experimenttally determined, is considered complex, we can easily guess analytical form of $G^{0}\left(t, t^{\prime}\right)$ can not be obtainable and numerical expression of $G^{0}\left(t, t^{\prime}\right)$ is only possible. Additionally analytical procedures to treat the term $\Delta(x)$ in Equation (1.19) is very difficult except for a few cases. Usually the assumption of $V_{\boldsymbol{k}}^{0}=V^{0}$ ( $\boldsymbol{k}$ independence) is strongly required, thus Equation (1.19) is

$$
\begin{aligned}
\Delta(x) & =\pi \sum_{k}\left|V_{k}^{0}\right|^{2} \delta\left(x-E_{k}\right)=\pi\left|V^{0}\right|^{2} \sum_{k} \delta\left(x-E_{k}\right) \\
& =\pi\left|V^{0}\right|^{2} \rho(x)
\end{aligned}
$$

In the above equation, $\rho(E)$ is D.O.S (Density of States) for conduction electrons. The assumption of $V_{k}^{0}=V^{0} \quad(\boldsymbol{k}$ independence $)$ is a very rough approximation and actual the systems seem to have $\boldsymbol{k}$ dependence, thus this approximation may not match the analysis of experimental data. Next, let us discuss the term of self energy $\Sigma\left(t_{1}, t_{2}\right)$. By substituting Equation (1.22) into Equation (1.18), we obtain following expression for self energy:

$$
\begin{aligned}
\Sigma\left(t_{1}, t_{2}\right)= & -\mathrm{i} \theta\left(t_{1}-t_{2}\right) \mu\left(t_{1}\right) \mu\left(t_{2}\right)\left|V^{0}\right|^{2} \\
& \times \int_{-\infty}^{\infty} \mathrm{d} x \rho(x) \exp \left(-\mathrm{i}\left(t_{1}-t_{2}\right) x / \hbar\right)
\end{aligned}
$$

Usually the term of $\Sigma\left(t_{1}, t_{2}\right)$ of Equation (1.23) can not be evaluated in the analytical form except for the specific cases such as $\rho(E)=\rho_{0}$ (const) or $\rho(E)=$ $\rho_{0} \delta\left(E-E_{0}\right)$, therefore, the procedure on the basis of (1.12) seems too complicated even if perturbative method is used. Consequently awkward and complex analytical and/or numerical schems for calculating integral parts and a lot of approximations ignoring experimental condi- tions are essentially required to evaluate $n(\infty)$. Actually, theoretical solutions are obatined in the limited conditions such as wide band limit.

Furthermore, if there exists impurity atoms or dislocations on surface, we should take account of the presence of impurity potential $\hat{H}^{\prime}$ as shown below

$$
\hat{H}^{\prime}=\sum_{k k^{\prime}} V_{k k^{\prime}} \hat{C}_{k}^{\dagger} \hat{C}_{k^{\prime}}
$$

When such a perturbative Hamiltonian is intoroduced into the system, it should be noted that the methods on the basis of Keldysh formalism seems almost impossible for evaluating $n(\infty)$ precisely because of a lots of awkward analytical and/or numerical tasks and approximations ignoring experimental conditions.

Concerning the other approaches, we can mention the works performed by Brako and Newns, [3,4]. Starting from the equation of motion method proposed by Bloss and Hone [5], they performed calculations by solving the simultaneous differential equations while regarding operators ( $Q$-number) as $c$-number. Based on these approaches, their method are expressed in the integral forms and exact solutions can be attainable in the case of wide band limit with assuming $\Delta(x) \cong$ constant. Actually experimental data on the polarisation of the light emitted in the electronic transition of $\mathrm{H}$ atoms scattered on $\mathrm{Ni}(111)$ surface [6] were theoretically interpreted and examined by their method [7].

However, their method also seems to meet the numerical and analytical difficulties and nuisances when perturbative Hamiltonian is introduced or the system shows complicated time-dependence because of integral schemes being involved. Actually, their method can not be applied to the case when impurity potential as shown in Equation (1.24) is introduced into the system.

Concerning these theoretical methods as stated above, the main reason for complicating calculations is that theoretical expressions are given not in the differential forms but in the integral ones. In this article, therefore, for the purpose of constructing the method which can be applicable to experimental analysis, we propose a new numerical methods on the basis of differential forms. At first we derive the extension of Heisenberg equation of motion to finite temperature. Then, based on the such an extension, we construct simultaneous differential equations which can evaluate time-dependent physical properties $\langle\hat{B}(t)\rangle_{\mathrm{av}}$ in finite temperature. Our method can be applied to the case when a perturbative Hamiltonian can show so complex time-dependent behaviours that analysis on the basis of linear response theory and/or of Green function methods seem to meet analytical difficulties and nuisance and even when perturbative Hamiltonian is newly introduced. 
In Section 2, we show the derivation of theoretical formalism and verify our proposed method for two cases: superconductivity and electrical conductivity. Furthermore we refer to the application to the many body problem using Hatree Fock approximation. After confirming our calculations, in Section 3, we apply our method to the two cases of resonant charge transfer processes, in which time-dependent properties play important roles on deciding dynamics. The first case is the most simlified resonant charge transfer process that a single charged ion is neutralzed in front of metal. During calculating, to simplify the discussion we assume electron transfer matrix remains constant. However it is sufficiently possible to estimate physical properties under the condition that electron transfer matrix shows $\boldsymbol{k}$ dependence. In the second case, we numerically treat the same process when impurity potential is introduced as perturbative Hamiltonian. The second case is more complicated than the first one and is usually considered as a difficult and awkward task to analyze on the basis of conventional ways such as Keldysh formalism because perturbative Hamiltonian often complicates the scheme of the evaluation of self energy. Furthermore, temperature dependence of resonant charge transfer process under the presence of impurity potential remains unresolved. Finally, in Section 4 we conclude this paper and discuss the remaining problems and further developments.

\section{Theoretical Approach, Its Verifications and Application to Many Body Problem}

\subsection{Derivation of Theoretical Framework}

Firstly, let us start from the Neumann equation, which is given by

$$
\mathrm{i} \hbar \frac{\partial}{\partial t} \hat{\rho}(t)=[\hat{H}, \hat{\rho}(t)],
$$

where operator $\hat{\rho}(t)$ is a time-dependent density operator. Additionally we set $\mu_{0}$ (chemical potential) $=0$ hereafter for simplifying the discussion. Then time-dependent physical properties $\langle\hat{B}(t)\rangle_{\mathrm{av}}$ in finite temperature is given by

$$
\langle\hat{B}(t)\rangle_{\mathrm{av}} \equiv \operatorname{Tr}[\hat{\rho}(t) \hat{B}] .
$$

By taking the the differentiation of the above equation, we obtain

$$
\frac{\mathrm{d}}{\mathrm{d} t}\langle\hat{B}(t)\rangle_{\mathrm{av}}=\frac{\mathrm{d}}{\mathrm{d} t} \operatorname{Tr}[\hat{\rho}(t) \hat{B}]=\operatorname{Tr}\left[\frac{\partial}{\partial t} \hat{\rho}(t) \hat{B}\right]
$$

Substituting the Neumann equation of Equation (2.1) into the above equation, we get

$$
\frac{\mathrm{d}}{\mathrm{d} t}\langle\hat{B}(t)\rangle_{\mathrm{av}}=\frac{1}{\mathrm{i} \hbar} \operatorname{Tr}[[\hat{H}, \rho(t)] \hat{B}] .
$$

Finally, using the relation $\operatorname{Tr}[\hat{X} \hat{Y}]=\operatorname{Tr}[\hat{Y} \hat{X}]$, we obtain the following formula with respect to the the differential form of $\langle\hat{B}(t)\rangle_{\mathrm{av}}$

$$
\begin{aligned}
\frac{\mathrm{d}}{\mathrm{d} t}\langle\hat{B}(t)\rangle_{\mathrm{av}} & =\frac{1}{\mathrm{i} \hbar} \operatorname{Tr}[\hat{\rho}(t)[\hat{B}, \hat{H}]] \\
& =\frac{1}{\mathrm{i} \hbar}\langle[\hat{B}, \hat{H}](t)\rangle_{\mathrm{av}}
\end{aligned}
$$

It should be easily noticed that Equation (2.5) takes the same form as Heisenberg equation of motion in Equation (1.1) except for the $c$-number. The above equation corresponds to the extension of Heisenberg equation of motion to finite temperature. Equation (2.5) directly leads to the fact that time-dependent physical properties $\langle\hat{B}(t)\rangle_{\mathrm{av}}$ in finite temperature are expressed not in the integral forms as Dyson equations but in the differential forms (simultaneous differetial equations); this, therefore, indicates analytical and/or numerical nuisances will be expected to greatly decrease even if Hamiltonian includes complex terms and/or perturbative Hamiltonian is introduced.

\subsection{Derivation of Gap Equation in Superconductivity}

Next, in order to verify the above theoretical results, let us apply the above equation to the superconductivity. we consider the following Hamiltonian:

$$
\begin{aligned}
\hat{K} & =\hat{H}-\mu_{0} \hat{N} \\
& =\sum_{\boldsymbol{k} \sigma} \xi_{\boldsymbol{k}} \hat{C}_{\boldsymbol{k} \sigma}^{\dagger} \hat{C}_{\boldsymbol{k} \sigma}+\sum_{\boldsymbol{k}, \boldsymbol{k}^{\prime}} V_{\boldsymbol{k}^{\prime} \boldsymbol{k}}(t) \hat{C}_{-\boldsymbol{k}^{\prime} \downarrow}^{\dagger} \hat{C}_{\boldsymbol{k}^{\prime} \uparrow}^{\dagger} \hat{C}_{\boldsymbol{k} \uparrow} \hat{C}_{-\boldsymbol{k} \downarrow},
\end{aligned}
$$

where

$$
\xi_{k}=E_{k}-\mu_{0}
$$

and

$$
V_{\boldsymbol{k}^{\prime} \boldsymbol{k}}(t)=\lambda(t) V_{\boldsymbol{k}^{\prime} \boldsymbol{k}}
$$

In the above equations, $E_{k}$ is the energy of a conduction electron with momentum $\boldsymbol{k} . \hat{C}_{\boldsymbol{k} \sigma}^{\dagger}$ and $\hat{C}_{\boldsymbol{k} \sigma}$ are creation and annihilation operators of a conduction electron with momentum $\boldsymbol{k}$ and spin orientations $\sigma$. Concerning $\lambda(t)$, this function increases monotonically and satisfies

$$
\lambda(-\infty)=0 \text { and } \lambda(\infty)=1
$$

Furthermore we introduce gap function $\Delta(\boldsymbol{k}, t)$ as defined below

$$
\Delta(\boldsymbol{k}, t)=-\sum_{\boldsymbol{q}} V_{\boldsymbol{k} \boldsymbol{q}}(t)\left\langle\hat{C}_{\boldsymbol{q}_{\uparrow}} \hat{C}_{-\boldsymbol{q} \downarrow}(t)\right\rangle_{\mathrm{av}} .
$$

Using mean field approximation and Equation (2.10), 
Hamiltonian Equation (2.6) can be reduced to oneelectron picture and given by

$$
\begin{aligned}
\hat{K}= & \sum_{\boldsymbol{k} \sigma} \xi_{\boldsymbol{k}} \hat{C}_{\boldsymbol{k} \sigma}^{\dagger} \hat{C}_{\boldsymbol{k} \sigma} \\
& -\sum_{\boldsymbol{k}}\left\{\Delta(\boldsymbol{k}, t) \hat{C}_{-\boldsymbol{k} \downarrow}^{\dagger} \hat{C}_{\boldsymbol{k} \uparrow}^{\dagger}+\Delta^{*}(\boldsymbol{k}, t) \hat{C}_{\boldsymbol{k} \uparrow} \hat{C}_{-\boldsymbol{k} \downarrow}\right\} \\
& +\sum_{\boldsymbol{k}} \Delta(\boldsymbol{k}, t)\left\langle\hat{C}_{-\boldsymbol{k} \downarrow}^{\dagger} \hat{C}_{\boldsymbol{k} \uparrow}^{\dagger}(t)\right\rangle_{\mathrm{av}} .
\end{aligned}
$$

Next, we take operator $\hat{B}$ as

$$
\hat{B}=\hat{C}_{q_{\uparrow}} \hat{C}_{-q \downarrow}
$$

Accordingly, $[\hat{B}, \hat{K}]$ is

$$
\begin{aligned}
{[\hat{B}, \hat{K}]=} & \xi_{-\boldsymbol{q}} \hat{C}_{\boldsymbol{q} \uparrow} \hat{C}_{-\boldsymbol{q} \downarrow}-\xi_{\boldsymbol{q}} \hat{C}_{-\boldsymbol{q} \downarrow} \hat{C}_{\boldsymbol{q} \uparrow} \\
& -\Delta(\boldsymbol{q}, t)\left(\hat{C}_{\boldsymbol{q} \uparrow} \hat{C}_{\boldsymbol{q}^{\dagger}}^{\dagger}-\hat{C}_{-\boldsymbol{q} \downarrow}^{\dagger} \hat{C}_{-\boldsymbol{q} \downarrow}\right) .
\end{aligned}
$$

Then, based on the Equation (2.5), the differential form of $\langle\hat{B}(t)\rangle_{\mathrm{av}}$ is

$$
\begin{aligned}
& \frac{\mathrm{d}}{\mathrm{d} t}\left\langle\hat{C}_{\boldsymbol{q}_{\uparrow}} \hat{C}_{-\boldsymbol{q} \downarrow}(t)\right\rangle_{\mathrm{av}} \\
& =\frac{1}{\mathrm{i} \hbar}\left\{\xi_{-\boldsymbol{q}}\left\langle\hat{C}_{\boldsymbol{q}^{\uparrow}} \hat{C}_{-\boldsymbol{q} \downarrow}(t)\right\rangle_{\mathrm{av}}-\xi_{\boldsymbol{q}}\left\langle\hat{C}_{-\boldsymbol{q} \downarrow} \hat{C}_{\boldsymbol{q} \uparrow}(t)\right\rangle_{\mathrm{av}}\right. \\
& \left.\quad-\Delta(\boldsymbol{q}, t)\left(\left\langle\hat{C}_{\boldsymbol{q}_{\uparrow}} \hat{C}_{\boldsymbol{q}^{\uparrow}}^{\dagger}(t)\right\rangle_{\mathrm{av}}-\left\langle\hat{C}_{-\boldsymbol{q} \downarrow}^{\dagger} \hat{C}_{-\boldsymbol{q} \downarrow}(t)\right\rangle_{\mathrm{av}}\right)\right\} .
\end{aligned}
$$

In the limit of $t \rightarrow \infty$,

$$
\begin{aligned}
& \frac{\mathrm{d}}{\mathrm{d} t}\left\langle\hat{C}_{\boldsymbol{q}_{\uparrow}} \hat{C}_{-\boldsymbol{q} \downarrow}(t)\right\rangle_{\mathrm{av}} \rightarrow 0 \\
& \Delta(\boldsymbol{q}, t) \rightarrow \Delta(\boldsymbol{q})(\because \lambda(\infty)=1)
\end{aligned}
$$

Consequently from Equation (2.14)

$$
\begin{aligned}
& 2 \xi_{\boldsymbol{q}}\left\langle\hat{C}_{\boldsymbol{q}_{\uparrow}} \hat{C}_{-\boldsymbol{q} \downarrow}(\infty)\right\rangle_{\mathrm{av}} \\
& =\Delta(\boldsymbol{q})\left(1-n_{\boldsymbol{q}_{\uparrow}}(\infty)-n_{-\boldsymbol{q} \downarrow}(\infty)\right)
\end{aligned}
$$

where

$$
\begin{aligned}
& n_{q_{\uparrow}}(\infty) \equiv\left\langle\hat{C}_{q \uparrow}^{\dagger} \hat{C}_{q_{\uparrow}}(\infty)\right\rangle_{\mathrm{av}} \\
& n_{-q \downarrow}(\infty) \equiv\left\langle\hat{C}_{-q \downarrow}^{\dagger} \hat{C}_{-q \downarrow}(\infty)\right\rangle_{\mathrm{av}}
\end{aligned}
$$

By combining the above relation with Equation (2.10), we obtain

$$
\Delta(\boldsymbol{k})=-\sum_{\boldsymbol{q}} \frac{V_{\boldsymbol{k} \boldsymbol{q}}}{2 \xi_{\boldsymbol{q}}} \Delta(\boldsymbol{q})\left(1-n_{\boldsymbol{q}_{\uparrow}}(\infty)-n_{-\boldsymbol{q} \downarrow}(\infty)\right)
$$

Next, using Bogoliubov transformation to the Hamiltonian of Equation (2.11) in the limit of $t \rightarrow \infty$

$$
\begin{aligned}
& \hat{C}_{\boldsymbol{k} \uparrow}=u_{\boldsymbol{k}} \hat{\alpha}_{\boldsymbol{k} \uparrow}+v_{\boldsymbol{k}} \hat{\alpha}_{-\boldsymbol{k} \downarrow}^{\dagger}, \\
& \hat{C}_{\boldsymbol{k} \downarrow}=u_{\boldsymbol{k}} \hat{\alpha}_{\boldsymbol{k} \downarrow}-v_{\boldsymbol{k}} \hat{\alpha}_{-\boldsymbol{k} \uparrow}^{\dagger},
\end{aligned}
$$

where $u_{k}$ and $v_{k}$ satisfies

$$
u_{k}^{2}+\left|v_{k}\right|^{2}=1 \text {. }
$$

Then Hamiltonian can be easily diagonalized and is given by

$$
\begin{aligned}
\hat{K}= & \sum_{\boldsymbol{k}} \tilde{E}_{k}\left\{\hat{\alpha}_{\boldsymbol{k} \uparrow}^{\dagger} \hat{\alpha}_{\boldsymbol{k} \uparrow}+\hat{\alpha}_{\boldsymbol{k} \downarrow}^{\dagger} \hat{\alpha}_{\boldsymbol{k} \downarrow}\right\} \\
& +\sum_{\boldsymbol{k}}\left\{2 \xi_{\boldsymbol{k}}\left|v_{\boldsymbol{k}}\right|^{2}-2 u_{\boldsymbol{k}}\left|v_{\boldsymbol{k}}\right| \cdot|\Delta(\boldsymbol{k})|\right\} \\
& +\sum_{\boldsymbol{k}} \Delta(\boldsymbol{k})\left\langle\hat{C}_{-\boldsymbol{k} \downarrow}^{\dagger} \hat{C}_{\boldsymbol{k} \uparrow}^{\dagger}\right\rangle_{\mathrm{av} .}
\end{aligned}
$$

where

$$
\tilde{E}_{\boldsymbol{k}} \equiv \sqrt{\xi_{\boldsymbol{k}}^{2}+|\Delta(\boldsymbol{k})|^{2}}
$$

From Bogoliubov transformation of Equation (2.19),

$$
\begin{aligned}
n_{\boldsymbol{q}_{\uparrow}}(\infty) & \equiv\left\langle\hat{C}_{\boldsymbol{q}_{\uparrow}}^{\dagger} \hat{C}_{\boldsymbol{q}_{\uparrow}}(\infty)\right\rangle_{\mathrm{av}}=\left\langle\hat{C}_{\boldsymbol{q}^{\uparrow}}^{\dagger} \hat{C}_{\boldsymbol{q}_{\uparrow}}\right\rangle_{\mathrm{av}} \\
& =u_{\boldsymbol{q}}^{2}\left\langle\hat{\alpha}_{q^{\uparrow}}^{\dagger} \hat{\alpha}_{\boldsymbol{q} \uparrow}\right\rangle_{\mathrm{av}}+\left|v_{\boldsymbol{q}}\right|^{2}\left(1-\left\langle\hat{\alpha}_{-\boldsymbol{q} \downarrow}^{\dagger} \hat{\alpha}_{-\boldsymbol{q} \downarrow}\right\rangle_{\mathrm{av}}\right) \\
n_{-\boldsymbol{q} \downarrow}(\infty) & \equiv\left\langle\hat{C}_{-\boldsymbol{q} \downarrow}^{\dagger} \hat{C}_{-\boldsymbol{q} \downarrow}(\infty)\right\rangle_{\mathrm{av}}=\left\langle\hat{C}_{-\boldsymbol{q} \downarrow}^{\dagger} \hat{C}_{-\boldsymbol{q} \downarrow}\right\rangle_{\mathrm{av}} \\
& =u_{\boldsymbol{q}}^{2}\left\langle\hat{\alpha}_{-\boldsymbol{q} \downarrow}^{\dagger} \hat{\alpha}_{-\boldsymbol{q} \downarrow}\right\rangle_{\mathrm{av}}+\left|v_{\boldsymbol{q}}\right|^{2}\left(1-\left\langle\hat{\alpha}_{\boldsymbol{q} \uparrow}^{\dagger} \hat{\alpha}_{q_{\uparrow} \uparrow}\right\rangle_{\mathrm{av}}\right)
\end{aligned}
$$

Since Hamiltonian is diagonalized as shown in Equation (2.21), we can easily estimate $\left\langle\hat{\alpha}_{q_{\uparrow}^{\dagger}}^{\dagger} \hat{\alpha}_{q_{\uparrow}}\right\rangle_{\mathrm{av}}$ and $\left\langle\hat{\alpha}_{-q \downarrow}^{\dagger} \hat{\alpha}_{-q \downarrow}\right\rangle_{\mathrm{av}}$. Then

$$
\left\langle\hat{\alpha}_{q \uparrow}^{\dagger} \hat{\alpha}_{q \uparrow}\right\rangle_{\mathrm{av}}=\left\langle\hat{\alpha}_{-q \downarrow}^{\dagger} \hat{\alpha}_{-q \downarrow}\right\rangle_{\mathrm{av}}=f_{e l}\left(\beta \tilde{E}_{q}\right),
$$

where $f_{e l}(x)$ is a Fermi-Dirac function $\left(f_{e l}(x) \equiv 1 /(1+\exp (x))\right)$. Consequently, the term of $1-n_{q_{\uparrow}}(\infty)-n_{-q \downarrow}(\infty)$ is

$$
1-n_{q \uparrow}(\infty)-n_{-q \downarrow}(\infty)=\frac{\xi_{q}}{\tilde{E}_{q}} \tanh \left(\frac{1}{2} \beta \tilde{E}_{q}\right) .
$$

By substituting the above result into Equation (2.18), we finally obtain gap equation.

$$
\Delta(\boldsymbol{k})=-\sum_{\boldsymbol{q}} \frac{V_{\boldsymbol{k} \boldsymbol{q}}}{2 \tilde{E}_{\boldsymbol{q}}} \Delta(\boldsymbol{q}) \tanh \left(\frac{1}{2} \beta \tilde{E}_{\boldsymbol{q}}\right) .
$$

\subsection{Calculation of Electrical Conductivity}

Next, we apply the Equation (2.5) to the electrical conduction. At first, we assume the following Hamiltonian:

$$
\begin{aligned}
& \hat{H}=\hat{H}_{0}+\hat{H}_{1} \\
& \hat{H}_{0}=\sum_{j=1}^{N} \frac{1}{2 m_{j}}\left\{\hat{\boldsymbol{P}}_{j}-\frac{Q_{j}}{c} \boldsymbol{A}\left(\boldsymbol{r}_{j}\right)\right\}^{2}+V\left(\boldsymbol{r}_{1}, \boldsymbol{r}_{2}, \cdots, \boldsymbol{r}_{N}\right) \\
& \hat{H}_{1}=-f(t) \sum_{j=1}^{N} Q_{j} \boldsymbol{r}_{j} \cdot \boldsymbol{E}
\end{aligned}
$$


where $m_{j}$ and $Q_{j}$ are $j$ th mass and charge of particles respectively, $c$ is a light velocity, and $V\left(\boldsymbol{r}_{1}, \boldsymbol{r}_{2}, \cdots \boldsymbol{r}_{N}\right)$ is a potential term. Magnetic Field $\boldsymbol{H}\left(\boldsymbol{r}_{j}\right)$ is expressed by using vector potential $\boldsymbol{A}\left(\boldsymbol{r}_{j}\right)$, i.e., $\boldsymbol{H}\left(\boldsymbol{r}_{j}\right)=\operatorname{rot} \boldsymbol{A}\left(\boldsymbol{r}_{j}\right)$. Electric field $\boldsymbol{E}$, vector of location $\boldsymbol{r}_{j}$ and $\hat{\boldsymbol{P}}_{j}$ are

$$
\begin{aligned}
\boldsymbol{E} & =\left(E_{x}, E_{y}, E_{z}\right)=\left(E_{1}, E_{2}, E_{3}\right), \\
\boldsymbol{r}_{j} & =\left(x_{j}, y_{j}, z_{j}\right)=\left(x_{j 1}, x_{j 2}, x_{j 3}\right), \\
\hat{\boldsymbol{P}}_{j} & =\left(\hat{P}_{j 1}, \hat{P}_{j 2}, \hat{P}_{j 3}\right)=-\mathrm{i} \hbar \nabla_{j} \\
& =-\mathrm{i} \hbar\left(\frac{\partial}{\partial x_{j 1}}, \frac{\partial}{\partial x_{j 2}}, \frac{\partial}{\partial x_{j 3}}\right)
\end{aligned}
$$

We obtain $\dot{x}_{j \mu}(\mu=1,2,3)$, the time differential form of $x_{j \mu}$, from the relation

$$
\begin{aligned}
& \mathrm{i} \hbar \dot{x}_{j \mu}=\left[x_{j \mu}, \hat{H}\right]=\left[x_{j \mu}, \hat{H}_{0}\right], \\
& \dot{x}_{j \mu}=\frac{1}{m_{j}}\left(\hat{P}_{j \mu}-\frac{Q_{j}}{c} \boldsymbol{A}\left(\boldsymbol{r}_{j}\right)\right)
\end{aligned}
$$

where

$$
\boldsymbol{A}\left(\boldsymbol{r}_{j}\right)=\left(A_{1}\left(\boldsymbol{r}_{j}\right), A_{2}\left(\boldsymbol{r}_{j}\right), A_{3}\left(\boldsymbol{r}_{j}\right)\right) .
$$

Since we focus on the electrical conduction, we take operator $\hat{B}$ as an electric current density along $\mu$ direction,

$$
\hat{B}=\hat{j}_{\mu}=\frac{1}{\Omega} \sum_{k=1}^{N} Q_{k} \dot{x}_{k \mu},
$$

where $\Omega$ is a volume of the system. Let us consider the case of magnetic field $\boldsymbol{H}(\boldsymbol{r})=0$, i.e., $\boldsymbol{A}(\boldsymbol{r})=0$, then we obtain the following from Equations (2.27) and (2.29),

$$
\begin{aligned}
{\left[\hat{j}_{\mu}, \hat{H}_{0}\right] } & =\frac{\hbar}{\mathrm{i} \Omega} \sum_{k=1}^{N} \frac{Q_{k}}{m_{k}}\left(\frac{\partial}{\partial x_{k \mu}} V\left(\boldsymbol{r}_{1} \cdots \boldsymbol{r}_{N}\right)\right), \\
{\left[\hat{j}_{\mu}, \hat{H}_{1}\right] } & =-\frac{f(t)}{\Omega} \sum_{k=1}^{N} \sum_{l=1}^{N} Q_{k} Q_{l}\left[\dot{x}_{k \mu}, \boldsymbol{r}_{l} \cdot \boldsymbol{E}\right] \\
& =-\frac{f(t)}{\Omega} \sum_{k=1}^{N} \sum_{l=1}^{N} \sum_{v=1}^{3} Q_{k} Q_{l} E_{v}\left[\dot{x}_{k \mu}, x_{l v}\right] .
\end{aligned}
$$

Using the relation of $\left[\dot{x}_{k \mu}, x_{l v}\right]=-\mathrm{i} \hbar \delta_{k l} \delta_{\mu v} / m_{k}$, $\left[\hat{j}_{\mu}, \hat{H}\right]$ is

$$
\begin{aligned}
{\left[\hat{j}_{\mu}, \hat{H}\right]=} & -\frac{\mathrm{i} \hbar}{\Omega} \sum_{k=1}^{N} \frac{Q_{k}}{m_{k}}\left(\frac{\partial}{\partial x_{k \mu}} V\left(\boldsymbol{r}_{1} \cdots \boldsymbol{r}_{N}\right)\right) \\
& +\frac{\mathrm{i} \hbar}{\Omega} f(t) E_{\mu} \sum_{k=1}^{N} \frac{Q_{k}^{2}}{m_{k}} .
\end{aligned}
$$

By applying the above result to Equation (2.5), we obtain the following differential equation with respect to the electric current density along $\mu$ direction:

$$
\begin{aligned}
\frac{\mathrm{d}}{\mathrm{d} t}\left\langle\hat{j}_{\mu}(t)\right\rangle_{\mathrm{av}}= & -\frac{1}{\Omega} \sum_{k=1}^{N} \frac{Q_{k}}{m_{k}}\left\langle\frac{\partial}{\partial x_{k \mu}} V\left(r_{1} \cdots r_{N}\right)\right\rangle_{\mathrm{av}} \\
& +\frac{f(t)}{\Omega} E_{\mu} \sum_{k=1}^{N} \frac{Q_{k}^{2}}{m_{k}} .
\end{aligned}
$$

It should be noted that the first term in the right hand expresses the force related to the potential $V$ while the second expresses term the force related to the applied electrical field. For example, let us consider the simplest case where $m_{k}=m=$ mass of electron, $Q_{k}=-e=$ charge of electron and $f(t) \equiv 1$, then

$$
\hat{j}_{\mu}(t) \rightarrow-e \frac{N}{\Omega} v_{\mu}(t),
$$

where $v_{\mu}(t)$ is a velocity of electron along the $\mu$ direction. Consequently, Equation (2.34) is

$$
m \frac{\mathrm{d}}{\mathrm{d} t}\left\langle v_{\mu}(t)\right\rangle_{\mathrm{av}}=-\frac{1}{N} \sum_{k=1}^{N}\left\langle\frac{\partial}{\partial x_{k \mu}} V\right\rangle_{\mathrm{av}}-e E_{\mu} .
$$

Furthermore, assuming that $\partial V / \partial x_{k \mu}$ is only dependent upon $\mu$, we obtain

$$
m \frac{\mathrm{d}}{\mathrm{d} t}\langle v(t)\rangle_{\mathrm{av}}=\langle-\nabla V\rangle_{\mathrm{av}}-e E,
$$

where $v(t)=\left(v_{x}(t), v_{y}(t), v_{z}(t)\right)$. In the above equation, the term of $-\nabla V$ indicates the force due to the potential $V$; thus Equation (2.37) corresponds to the classical equation of motion.

Next, let us back to the Equation (2.34). We solve this equation under the initial condition of $\left\langle\hat{j}_{\mu}(0)\right\rangle_{\mathrm{av}}=0$. The solution of Equation (2.34) can be easily evaluated as below

$$
\begin{aligned}
\left\langle\hat{j}_{\mu}(t)\right\rangle_{\mathrm{av}}= & -\frac{1}{\Omega} \sum_{k=1}^{N} \frac{Q_{k}}{m_{k}} \int_{0}^{t}\left\langle\frac{\partial}{\partial x_{k \mu}} V\left(\boldsymbol{r}_{1} \cdots \boldsymbol{r}_{N}\right)\right\rangle_{\mathrm{av}} \mathrm{d} \tau \\
& +\frac{E_{\mu}}{\Omega} \sum_{k=1}^{N} \frac{Q_{k}^{2}}{m_{k}} \int_{0}^{t} f(\tau) \mathrm{d} \tau
\end{aligned}
$$

If we focus on the term of $\left\langle\partial V / \partial x_{k \mu}\right\rangle_{\mathrm{av}}$ in the right hand of the above equation, the exact expression of this term is:

$$
\left\langle\frac{\partial}{\partial x_{k \mu}} V\left(\boldsymbol{r}_{1} \cdots \boldsymbol{r}_{N}\right)\right\rangle_{\mathrm{av}}=\operatorname{Tr}\left\{\hat{\rho}(\tau) \frac{\partial}{\partial x_{k \mu}} V\left(\boldsymbol{r}_{1} \cdots \boldsymbol{r}_{N}\right)\right\} .
$$

The density operator $\hat{\rho}(\tau)$ of Equation (2.39) can be extended into the series of the power of $f(\tau)$.

$$
\hat{\rho}(\tau)=\hat{\rho}_{0}+f(\tau) \hat{\Lambda}_{1}+f(\tau)^{2} \hat{\Lambda}_{2}+\cdots .
$$

Consequently,using the above extension of $\hat{\rho}(\tau)$, we obtain the following result when $f(\tau)=\exp (-\mathrm{i} \omega \tau)$ : 


$$
\begin{aligned}
& \int_{0}^{t}\left\langle\frac{\partial V}{\partial x_{k \mu}}\right\rangle_{\mathrm{av}} \mathrm{d} \tau \\
& =t \operatorname{Tr}\left\{\hat{\rho}_{0} \frac{\partial V}{\partial x_{k \mu}}\right\}+\frac{\mathrm{i}}{\omega} \sum_{l=1}^{\infty} \operatorname{Tr}\left\{\hat{\Lambda}_{l} \frac{\partial V}{\partial x_{k \mu}}\right\} \frac{\exp (-\mathrm{i} l \omega t)-1}{l}
\end{aligned}
$$

$\left\langle\hat{j}_{\mu}(t)\right\rangle_{\text {av }}$ is calculated by substituting Equation (2.41) into Equation (2.38),

$$
\begin{aligned}
\left\langle\hat{j}_{\mu}(t)\right\rangle_{\mathrm{av}}= & -\frac{t}{\Omega} \sum_{k=1}^{N} \frac{Q_{k}}{m_{k}} \operatorname{Tr}\left\{\hat{\rho}_{0} \frac{\partial V}{\partial x_{k \mu}}\right\} \\
& -\frac{\mathrm{i}}{\Omega \omega} \sum_{k=1}^{N} \frac{Q_{k}}{m_{k}} \sum_{l=1}^{\infty} \operatorname{Tr}\left\{\hat{\Lambda}_{l} \frac{\partial V}{\partial x_{k \mu}}\right\} \frac{\exp (-\mathrm{i} l \omega t)-1}{l} \\
& +\frac{\mathrm{i} E_{\mu}}{\Omega \omega}(\exp (-\mathrm{i} \omega t)-1) \sum_{k=1}^{N} \frac{Q_{k}^{2}}{m_{k}}
\end{aligned}
$$

Considering the case where there is no pertubative Hamiltonian of $\hat{H}_{1}$, we can easily conclude no electrical current, which means $\left\langle\hat{j}_{\mu}(t)\right\rangle_{\mathrm{av}}=0$. Then

$$
\mathrm{i} \hbar \frac{\mathrm{d}}{\mathrm{d} t}\left\langle\hat{j}_{\mu}(t)\right\rangle_{\mathrm{av}}=\left\langle\left[\hat{j}_{\mu}, \hat{H}_{0}\right]\right\rangle_{\mathrm{av}}=0 .
$$

Consequently,

$$
\frac{\hbar}{\mathrm{i} \Omega} \sum_{k=1}^{N} \frac{Q_{k}}{m_{k}}\left\langle\frac{\partial V}{\partial x_{k \mu}}\right\rangle_{\text {av }}=\frac{\hbar}{\mathrm{i} \Omega} \sum_{k=1}^{N} \frac{Q_{k}}{m_{k}} \operatorname{Tr}\left\{\hat{\rho}_{0} \frac{\partial V}{\partial x_{k \mu}}\right\}=0 .
$$

From the above result, even if pertubative Hamiltonian of $\hat{H}_{1}$ exists, $\left\langle\hat{j}_{\mu}(t)\right\rangle_{\mathrm{av}}$ is

$$
\begin{aligned}
& \left\langle\hat{j}_{\mu}(t)\right\rangle_{\mathrm{av}} \\
& =-\frac{\mathrm{i}}{\Omega \omega} \sum_{k=1}^{N} \frac{Q_{k}}{m_{k}} \sum_{l=1}^{\infty} \operatorname{Tr}\left\{\hat{\Lambda}_{l} \frac{\partial V}{\partial x_{k \mu}}\right\} \frac{\exp (-\mathrm{i} l \omega t)-1}{l} \\
& +\frac{\mathrm{i} E_{\mu}}{\Omega \omega}(\exp (-\mathrm{i} \omega t)-1) \sum_{k=1}^{N} \frac{Q_{k}^{2}}{m_{k}} .
\end{aligned}
$$

Then,

$$
\lim _{\omega \rightarrow \infty}\left\langle\hat{j}_{\mu}(t)\right\rangle_{\mathrm{av}}=0
$$

Since $l$ in the Equation (2.44) corresponds to the power of Hamiltonian $\hat{H}_{1}$, it is sufficient to take $l=1$ within the range of linearity, i.e., the first order of Hamiltonian $\hat{H}_{1}$. Accordingly, on the basis of linear response theory, $\hat{\Lambda}_{1}$ is

$$
\hat{\Lambda}_{1}=\frac{\mathrm{i}}{\hbar} \int_{0}^{\infty} \mathrm{e}^{\mathrm{i} \omega \tau} \mathrm{e}^{-\mathrm{i} \hat{H}_{0} \tau / \hbar}\left[\hat{A}, \hat{\rho}_{0}\right] \mathrm{e}^{\mathrm{i} \hat{H}_{0} \tau / \hbar} \mathrm{d} \tau,
$$

where

$$
\hat{A}=\sum_{j=1}^{N} Q_{j} \boldsymbol{r}_{j} \cdot \boldsymbol{E} .
$$

Consequently, within the range of linearity, $\left\langle\hat{j}_{\mu}(t)\right\rangle_{\mathrm{av}}$ is expressed as below,

$$
\begin{aligned}
& \left\langle\hat{j}_{\mu}(t)\right\rangle_{\mathrm{av}}=-\frac{\mathrm{i}}{\Omega \omega}\left(\mathrm{e}^{-\mathrm{i} \omega t}-1\right) \\
& \times \sum_{k=1}^{N} \frac{Q_{k}}{m_{k}} \operatorname{Tr}\left[\frac{\mathrm{i}}{\hbar} \int_{0}^{\infty} \mathrm{d} \tau \mathrm{e}^{\mathrm{i} \omega \tau} \mathrm{e}^{-\mathrm{i} \hat{H}_{0} \tau / \hbar}\left[\hat{A}, \hat{\rho}_{0}\right] \mathrm{e}^{\mathrm{i} \hat{H}_{0} \tau / \hbar} \frac{\partial V}{\partial x_{k \mu}}\right] \\
& +\frac{\mathrm{i} E_{\mu}}{\Omega \omega}\left(\mathrm{e}^{-\mathrm{i} \omega t}-1\right) \sum_{k=1}^{N} \frac{Q_{k}^{2}}{m_{k}} .
\end{aligned}
$$

We define $\left\langle\hat{j}_{\mu}(t)\right\rangle_{\mathrm{av}}$ and electrical conductivity $\sigma_{\mu v}$ in the following manner:

$$
\begin{aligned}
& \left\langle\hat{j}_{\mu}(t)\right\rangle_{\mathrm{av}}=\left(\mathrm{e}^{-\mathrm{i} \omega t}-1\right) j_{\mu}(\omega), \\
& j_{\mu}(\omega)=\frac{1}{\Omega} \sum_{v} \sigma_{\mu \nu}(\omega) E_{v} .
\end{aligned}
$$

Furthermore

$$
\lim _{\omega \rightarrow \infty} \int_{0}^{\infty} \mathrm{d} \tau \mathrm{e}^{\mathrm{i} \omega \tau} \operatorname{Tr}\left\{\mathrm{e}^{-\mathrm{i} \hat{H}_{0} \tau / \hbar}\left[\hat{A}, \hat{\rho}_{0}\right] \mathrm{e}^{\mathrm{i} \hat{H}_{0} \tau / \hbar} \frac{\partial V}{\partial x_{k \mu}}\right\} \rightarrow 0 .
$$

Finally we obtain the sum rule

$$
\lim _{\omega \rightarrow \infty} \omega \sigma_{\mu \mu}(\omega) / i \rightarrow \sum_{k=1}^{N} \frac{Q_{k}^{2}}{m_{k}}
$$

\subsection{Application to Many Body Problem}

Concerning the application to many body problem, let us consider the follwing Anderson Hamiltonian:

$$
\begin{aligned}
\hat{H}= & \hat{H}_{0}+\hat{H}_{1}, \\
\hat{H}_{0}= & \sum_{\boldsymbol{k} \sigma} E_{\boldsymbol{k}} \hat{C}_{k \sigma}^{\dagger} \hat{C}_{k \sigma}+\sum_{\sigma} E_{d} \hat{C}_{d \sigma}^{\dagger} \hat{C}_{d \sigma} \\
& +\sum_{k \sigma}\left(V_{k d} \hat{C}_{k \sigma}^{\dagger} \hat{C}_{d \sigma}+V_{d k} \hat{C}_{d \sigma}^{\dagger} \hat{C}_{k \sigma}\right), \\
\hat{H}_{1}= & U \hat{C}_{d \uparrow}^{\dagger} \hat{C}_{d \uparrow} \hat{C}_{d \downarrow}^{\dagger} \hat{C}_{d \downarrow},
\end{aligned}
$$

$E_{k}, \hat{C}_{k \sigma}^{\dagger}, \hat{C}_{k \sigma}$ and $\sigma$ are the same as defined in sub section 2.2. $\hat{C}_{d \sigma}^{+}$and $\hat{C}_{d \sigma}$ are creation and anihilation operators of a $d$ electron with spin orientation $\sigma . E_{d}$ and $V_{\boldsymbol{k} d}$ denotes energy level of a $d$ electron and electron transfer matrix element from a $d$ electron to conduction electron with momentum $\boldsymbol{k}$, respectively. $U$ is a Coulomb repulsive energy between $d \uparrow$ and $d \downarrow$ electrons.

Going through the same procedures as shown in Sub Section 2.2, we obtain the following simultaneous differential equations: 


$$
\begin{aligned}
& \frac{\mathrm{d}}{\mathrm{d} t}\left\langle n_{d \sigma \boldsymbol{k} \sigma}(t)\right\rangle_{\mathrm{av}} \\
& =\frac{1}{\mathrm{i} \hbar}\left(E_{\boldsymbol{k}}-E_{d}\right)\left\langle n_{d \sigma \boldsymbol{k} \sigma}(t)\right\rangle_{\mathrm{av}} \\
& \quad-\frac{1}{\mathrm{i} \hbar} \sum_{q} V_{q d}\left\langle n_{q \sigma \boldsymbol{k} \sigma}(t)\right\rangle_{\mathrm{av}}+\frac{1}{\mathrm{i} \hbar} V_{\boldsymbol{k} d}\left\langle n_{d \sigma d \sigma}(t)\right\rangle_{\mathrm{av}} \\
& \quad-\frac{1}{\mathrm{i} \hbar} U\left\langle n_{d \bar{\sigma} d \bar{\sigma} d \sigma \boldsymbol{k} \sigma}(t)\right\rangle_{\mathrm{av}}, \\
& \frac{\mathrm{d}}{\mathrm{d} t}\left\langle n_{d \sigma d \sigma}(t)\right\rangle_{\mathrm{av}} \\
& =\frac{1}{\mathrm{i} \hbar} \sum_{q}\left(V_{d \boldsymbol{k}}\left\langle n_{d \sigma \boldsymbol{k} \sigma}(t)\right\rangle_{\mathrm{av}}-V_{\boldsymbol{k} d}\left\langle n_{\boldsymbol{k} \sigma d \sigma}(t)\right\rangle_{\mathrm{av}}\right), \\
& \frac{\mathrm{d}}{\mathrm{d} t}\left\langle n_{q \sigma \boldsymbol{k} \sigma}(t)\right\rangle_{\mathrm{av}} \\
& =\frac{1}{\mathrm{i} \hbar}\left(V_{\boldsymbol{k} d}\left\langle n_{q \sigma d \sigma}(t)\right\rangle_{\mathrm{av}}-V_{d q}\left\langle n_{d \sigma \boldsymbol{k} \sigma}(t)\right\rangle_{\mathrm{av}}\right. \\
& \left.\quad+\left(E_{\boldsymbol{k}}-E_{q}\right)\left\langle n_{q \sigma k \sigma}(t)\right\rangle_{\mathrm{av}}\right)
\end{aligned}
$$

where $\left\langle n_{i j}(t)\right\rangle_{\mathrm{av}} \equiv \operatorname{Tr}\left[\hat{\rho}(t) \hat{C}_{i}^{\dagger} \hat{C}_{j}\right]$ and $\left\langle n_{i j k l}(t)\right\rangle_{\mathrm{av}} \equiv \operatorname{Tr}\left[\hat{\rho}(t) \hat{C}_{i}^{\dagger} \hat{C}_{j} \hat{C}_{k}^{\dagger} \hat{C}_{l}\right]$. In the above equation, $\bar{\sigma}$ means $-\sigma$, e.g., $\bar{\sigma}=\downarrow$ if $\sigma=\uparrow$.

As illustrated in Equations (2.54)-(2.56), these simultaneous differential equations can not be numerically evaluated due to the prsesence of many body terms of $\left\langle n_{d \downarrow d \downarrow d \uparrow k \uparrow}(t)\right\rangle_{\mathrm{av}}$ and $\left\langle n_{d \uparrow^{\uparrow \uparrow} \uparrow_{\downarrow \downarrow k \downarrow}}(t)\right\rangle_{\mathrm{av}}$. For the purpose of solving these equations numerically, we introduce such an approximation as below,

$$
\left\langle n_{i j k l}(t)\right\rangle_{\mathrm{av}} \cong\left\langle n_{i j}(t)\right\rangle_{\mathrm{av}}\left\langle n_{k l}(t)\right\rangle_{\mathrm{av}} .
$$

Thus, $\left\langle n_{d \downarrow d \downarrow d \uparrow \uparrow \uparrow}(t)\right\rangle_{\text {av }}$ and $\left\langle n_{d \uparrow d^{\uparrow} d \downarrow k \downarrow}(t)\right\rangle_{\text {av }}$ are

$$
\begin{aligned}
& \left\langle n_{d \downarrow d \downarrow d \uparrow \boldsymbol{k} \uparrow}(t)\right\rangle_{\mathrm{av}} \cong\left\langle n_{d \downarrow d \downarrow}(t)\right\rangle_{\mathrm{av}}\left\langle n_{d \uparrow \boldsymbol{k} \uparrow}(t)\right\rangle_{\mathrm{av}}, \\
& \left\langle n_{d \uparrow d \uparrow d \downarrow k \downarrow}(t)\right\rangle_{\mathrm{av}} \cong\left\langle n_{d \uparrow d^{\uparrow}}(t)\right\rangle_{\mathrm{av}}\left\langle n_{d \downarrow \boldsymbol{k} \downarrow}(t)\right\rangle_{\mathrm{av}} .
\end{aligned}
$$

By substituting Equation (2.58) into Equation (2.54), non-linear simulataneous differential equations of Equation (2.59) to Equation (2.61) are deduced with showing the numerical solvability. thus we can evaluate using computer codes.

$$
\begin{aligned}
& \frac{\mathrm{d}}{\mathrm{d} t}\left\langle n_{d \sigma k \sigma}(t)\right\rangle_{\mathrm{av}} \\
& =\frac{1}{\mathrm{i} \hbar}\left(E_{\boldsymbol{k}}-E_{d}-U\left\langle n_{d \bar{\sigma} d \bar{\sigma}}(t)\right\rangle_{\mathrm{av}}\right) \\
& \times\left\langle n_{d \sigma k \sigma}(t)\right\rangle_{\mathrm{av}}-\frac{1}{\mathrm{i} \hbar} \sum_{q} V_{q d}\left\langle n_{q \sigma k \sigma}(t)\right\rangle_{\mathrm{av}} \\
& +\frac{1}{\mathrm{i} \hbar} V_{\boldsymbol{k} d}\left\langle n_{d \sigma d \sigma}(t)\right\rangle_{\mathrm{av}} .
\end{aligned}
$$

$$
\begin{aligned}
& \frac{\mathrm{d}}{\mathrm{d} t}\left\langle n_{d \sigma d \sigma}(t)\right\rangle_{\mathrm{av}} \\
& =\frac{1}{\mathrm{i} \hbar} \sum_{\boldsymbol{k}}\left(V_{d \boldsymbol{k}}\left\langle n_{d \sigma \boldsymbol{k} \sigma}(t)\right\rangle_{\mathrm{av}}-V_{\boldsymbol{k} d}\left\langle n_{\boldsymbol{k} \sigma d \sigma}(t)\right\rangle_{\mathrm{av}}\right) \\
& \frac{\mathrm{d}}{\mathrm{d} t}\left\langle n_{q \sigma k \sigma}(t)\right\rangle_{\mathrm{av}} \\
& =\frac{1}{\mathrm{i} \hbar}\left(V_{\boldsymbol{k} d}\left\langle n_{q \sigma d \sigma}(t)\right\rangle_{\mathrm{av}}-V_{d q}\left\langle n_{d \sigma \boldsymbol{k} \sigma}(t)\right\rangle_{\mathrm{av}}\right. \\
& \left.\quad+\left(E_{\boldsymbol{k}}-E_{q}\right)\left\langle n_{q \sigma \kappa \sigma}(t)\right\rangle_{\mathrm{av}}\right)
\end{aligned}
$$

It should be noted that our method is quite simple because these non-linear simulataneous differential equations do not have any terms related to comlicated selfenergies or vertex corrections, which should be essentially involved in considering the electrons correlation.

Next, let us discuss the above approximation in the view points of mean field approximation (Hatree Fock approximation). Firstly, we define $\hat{n}_{d \sigma}$ as

$$
\hat{n}_{d \sigma}=\hat{C}_{d \sigma}^{\dagger} \hat{C}_{d \sigma}=\left\langle\hat{n}_{d \sigma}\right\rangle+\left(n_{d \sigma}-\left\langle\hat{n}_{d \sigma}\right\rangle\right),
$$

where $\left\langle\hat{n}_{d \sigma}\right\rangle$ denotes average value of $\hat{n}_{d \sigma}$. Then, ignoring the $\left(n_{d \sigma}-\left\langle\hat{n}_{d \sigma}\right\rangle\right)\left(n_{d \bar{\sigma}}-\left\langle\hat{n}_{d \bar{\sigma}}\right\rangle\right)$, we obtain the following Hamiltonian by substituting the above equation into Coulomb repulsive term of Equation (2.53):

$$
\begin{aligned}
\hat{H}= & \sum_{\boldsymbol{k} \sigma} E_{\boldsymbol{k}} \hat{C}_{\boldsymbol{k} \sigma}^{\dagger} \hat{C}_{\boldsymbol{k} \sigma}+\sum_{\sigma}\left(E_{d}+U\left\langle\hat{n}_{d \bar{\sigma}}\right\rangle\right) \hat{C}_{d \sigma}^{\dagger} \hat{C}_{d \sigma} \\
& +\sum_{\boldsymbol{k} \sigma}\left(V_{\boldsymbol{k} d} \hat{C}_{\boldsymbol{k} \sigma}^{\dagger} \hat{C}_{d \sigma}+V_{d \boldsymbol{k}} \hat{C}_{d \sigma}^{\dagger} \hat{C}_{\boldsymbol{k} \sigma}\right) .
\end{aligned}
$$

From the above Hamiltonian, simultaneous the differential equations of Equations (2.64)-(2.66) are derived as the following:

$$
\begin{aligned}
& \frac{\mathrm{d}}{\mathrm{d} t}\left\langle n_{d \sigma k \sigma}(t)\right\rangle_{\mathrm{av}} \\
& =\frac{1}{\mathrm{i} \hbar}\left(E_{\boldsymbol{k}}-E_{d}-U\left\langle\hat{n}_{d \bar{\sigma}}\right\rangle\right)\left\langle n_{d \sigma k \sigma}(t)\right\rangle_{\mathrm{av}} \\
& \quad-\frac{1}{\mathrm{i} \hbar} \sum_{q} V_{q d}\left\langle n_{q \sigma k \sigma}(t)\right\rangle_{\mathrm{av}}+\frac{1}{\mathrm{i} \hbar} V_{k d}\left\langle n_{d \sigma d \sigma}(t)\right\rangle_{\mathrm{av}}, \\
& \frac{\mathrm{d}}{\mathrm{d} t}\left\langle n_{d \sigma d \sigma}(t)\right\rangle_{\mathrm{av}} \\
& =\frac{1}{\mathrm{i} \hbar} \sum_{k}\left(V_{d k}\left\langle n_{d \sigma k \sigma}(t)\right\rangle_{\mathrm{av}}-V_{\boldsymbol{k} d}\left\langle n_{k \sigma d \sigma}(t)\right\rangle_{\mathrm{av}}\right), \\
& \frac{\mathrm{d}}{\mathrm{d} t}\left\langle n_{q \sigma k \sigma}(t)\right\rangle_{\mathrm{av}} \\
& =\frac{1}{\mathrm{i} \hbar}\left(V_{k d}\left\langle n_{q \sigma d \sigma}(t)\right\rangle_{\mathrm{av}}-V_{d q}\left\langle n_{d \sigma k \sigma}(t)\right\rangle_{\mathrm{av}}\right. \\
& \left.\quad+\left(E_{\boldsymbol{k}}-E_{q}\right)\left\langle n_{q \sigma k \sigma}(t)\right\rangle_{\mathrm{av}}\right) .
\end{aligned}
$$

Since $\left\langle\hat{n}_{d \sigma}\right\rangle$ is the average value of $\hat{n}_{d \sigma}$, accord- 
ingly it seems resonable to replace $\left\langle\hat{n}_{d \bar{\sigma}}\right\rangle$ by $\left\langle n_{d \bar{\sigma} d \bar{\sigma}}(t)\right\rangle_{\text {av }}$. With the above replacement, Equation (2.64) turns out to be equivalent to Equation (2.59). Additionally one can easily see that Equation (2.65) and (2.66) are equivalent to (2.60) and (2.61), respectively. It can be, therefore, concluded that the approximation of Equation (2.58) is regarded as Hatree Fock approximation. In this article, we prefer to introduce Hartree Fock approximation into our proposed method.

\section{Application to the RCT Neutralization on Surface}

As firstly stated in the previous article "Analysis of Resonance Charge Transfer Neutralization on the Basis of Heisenberg Equations of Motion" [1], a resonant electron charge transfer (RCT) between a metal surface and an atom is interesting and important event, which Newns first proposed and discussed to explain the chemical adsorption in 1969 [8] on the basis of Anderson-Newns Model. In considering the various surface phenomena, RCT have played important roles to determine the surface dynamical processes such as neutralization, molecular dissociation and chemisorption. In the theoretical framework to treat RCT, many investigations related to the analysis on time-dependent Anderson-Newns model (TDAN) have been reported and discussed extensively to account for the various experimental results. The evaluation of time-dependent properties, therefore, are essential in analyzing various results related to such surface dynamical processes. Concerning to these analysis, it is well known that the Keldysh formalism [9] has been often used, because this method was developed for the purpose of being applicable to non-equilibrium states such as TDAN. Consequently analysis on the basis of the above formalism have been reported and discussed in the field of surface science [10-16].

In this section, firstly, let us apply our theoretical results to the most simplified RCT neutralization process, where a singly charged ion approaches a metal surface and moves away from it after the ion-surface collision. To describe the electronic interaction between a metal surface and an ion, we consider the following TDAN while ignoring spin orientations:

$$
\begin{aligned}
& \hat{H}=\hat{H}_{0}+\hat{H}_{1}, \\
& \hat{H}_{0}=\sum_{k} E_{k} \hat{C}_{k}^{\dagger} \hat{C}_{k}+E_{a}(z) \hat{C}_{a}^{\dagger} \hat{C}_{a}, \\
& \hat{H}_{1}=\sum_{k}\left(V_{a k}(z) \hat{C}_{a}^{\dagger} \hat{C}_{k}+V_{k a}(z) \hat{C}_{k}^{\dagger} \hat{C}_{a}\right) .
\end{aligned}
$$

In the above equations, all variables and operator are same as alleady defined in the section of introduction. Since $z$ can be expressed as a function of time $(z=v|t| v$ : velocity of ion), we use $E_{a}(t), V_{a k}(t)$ and $V_{k a}(t)$ instead of $E_{a}(z), V_{a k}(z)$ and $V_{k a}(z)$. We take $\hat{B}$ as

$$
\hat{B}=\hat{C}_{a}^{\dagger} \hat{C}_{a}, \hat{C}_{\boldsymbol{k}}^{\dagger} \hat{C}_{a}, \hat{C}_{\boldsymbol{k}}^{\dagger} \hat{C}_{\boldsymbol{k}^{\prime}}
$$

By combining Equations (3.1)-(3.4) with equation (2.5), we obtain

$$
\begin{aligned}
& \frac{\mathrm{d}}{\mathrm{d} t}\left\langle n_{a a}(t)\right\rangle_{\mathrm{av}} \\
&=\frac{\mathrm{i}}{\hbar} \sum_{k} V_{k a}(t)\left\langle n_{\boldsymbol{k} a}(t)\right\rangle_{\mathrm{av}}-\frac{\mathrm{i}}{\hbar} \sum_{\boldsymbol{k}} V_{a \boldsymbol{k}}(t)\left\langle n_{a k}(t)\right\rangle_{\mathrm{av}}, \\
& \frac{\mathrm{d}}{\mathrm{d} t}\left\langle n_{\boldsymbol{k} a}(t)\right\rangle_{\mathrm{av}}= \frac{\mathrm{i}}{\hbar}\left(E_{\boldsymbol{k}}-E_{a}(t)\right)\left\langle n_{\boldsymbol{k} a}(t)\right\rangle_{\mathrm{av}} \\
&+\frac{\mathrm{i}}{\hbar} V_{a \boldsymbol{k}}(t)\left\langle n_{a a}(t)\right\rangle_{\mathrm{av}} \\
&-\frac{\mathrm{i}}{\hbar} \sum_{\boldsymbol{k}^{\prime}} V_{a k^{\prime}}(t)\left\langle n_{\boldsymbol{k} k^{\prime}}(t)\right\rangle_{\mathrm{av}} \\
& \frac{\mathrm{d}}{\mathrm{d} t}\left\langle n_{\boldsymbol{k} k^{\prime}}(t)\right\rangle_{\mathrm{av}}= \frac{\mathrm{i}}{\hbar}\left(E_{\boldsymbol{k}}-E_{\boldsymbol{k}^{\prime}}\right)\left\langle n_{\boldsymbol{k} \boldsymbol{k}^{\prime}}(t)\right\rangle_{\mathrm{av}} \\
&+\frac{\mathrm{i}}{\hbar} V_{a \boldsymbol{k}}(t)\left\langle n_{a k^{\prime}}(t)\right\rangle_{\mathrm{av}} \\
&-\frac{\mathrm{i}}{\hbar} V_{\boldsymbol{k}^{\prime} a}(t)\left\langle n_{\boldsymbol{k} a}(t)\right\rangle_{\mathrm{av}} .
\end{aligned}
$$

where we define

$$
\left\langle n_{i j}(t)\right\rangle_{\mathrm{av}} \equiv \operatorname{Tr}\left[\hat{\rho}(t) \hat{C}_{i}^{\dagger} \hat{C}_{j}\right]
$$

and $\left\langle n_{j i}(t)\right\rangle_{a y}$ is obtained from the relation $\left\langle n_{j i}(t)\right\rangle_{\mathrm{av}}=\left(\left\langle n_{i j}(t)\right\rangle_{\mathrm{av}}\right) *$.

Consequently, we obtain the simultaneous differential equations corresponding to the Hamiltonian of Equation (3.1). It should be noted that evaluated differential equations are the same expressions as already stated in the previous article [1]. The differences between finite temperature case and ground state $(0 \mathrm{~K})$ are initial conditions. The merit of this method is that one can obtain numerical solutions of Equations (3.5)-(3.7) under various initial conditions by using computer codes. As stated in the introduction, since Equations (3.5)-(3.7) are given in the differential forms, numerical procedures toward the solutions are basically free from such nuisances as integral schemes ascribed to Dyson equation. Consequently our proposed method seems to be applicable to the cases even when Hamiltonian includes complicated time dependent terms.

When calculating these simultaneous differential equations, we follow the same procedures as stated in the previous article [1]. Concerning the surface-particle (ion) interaction, we adopt the Gaussian form of $V_{a k}(t)=V_{a}^{0} \exp \left(-\zeta v^{2} t^{2}\right) \quad(\zeta$ : constant, $v$ : ion velocity $)$ without an imaginary part and momentum $\boldsymbol{k}$ dependence. For the purpose of simplifying the discussion, we assume 
that $V_{a \boldsymbol{k}}(t)$ has $\boldsymbol{k}$ independence. However, as shown in Equations (3.5)-(3.7), it is sufficiently possible to calculate numerically in the case of $V_{a \boldsymbol{k}}(t)$ having $\boldsymbol{k}$ dependence. Simultaneous differential equations obtained from Equations (3.5)-(3.7) are solved numerically under the initial conditions of $\left\langle n_{a a}(-\infty)\right\rangle_{\mathrm{av}}=0$,

$\left\langle n_{k a}(-\infty)\right\rangle_{a v}=0$ and $\left\langle n_{k k^{\prime}}(-\infty)\right\rangle_{\mathrm{av}}=\delta_{k k^{\prime}} f_{\mathrm{el}}\left(\beta E_{\boldsymbol{k}}\right)$

where $\left\langle n_{\boldsymbol{k} \boldsymbol{k}}(-\infty)\right\rangle_{\mathrm{av}}$ and $\left\langle n_{a a}(-\infty)\right\rangle_{\mathrm{av}}$ correspond to the number of electrons occupying momentum $\boldsymbol{k}$ and the complete ionic state at $t \rightarrow-\infty$, respectively. We assume that Fermi level $\mu_{0}$ remains in the middle of the band and that bandwidth $D$ and energy interval $\Delta E$ are $0.8 \mathrm{eV}$ and $0.01 \mathrm{eV}$, respectively. The range of energy $E_{k}$ is $-0.4 \mathrm{eV} \leq E_{k} \leq 0.4 \mathrm{eV}$ and half of band, i.e., -0.4 $\mathrm{eV} \leq E_{k} \leq 0 \mathrm{eV}$ is filled with electrons at $T=0 \mathrm{~K}$.

Additionally, when the ion is close to the surface, the atomic level of the ion actually fluctuates because of frequent electron transfers between the surface and the ion. Thus, calculations including such a correction are required. In this article, we take the same form as stated in previous aricle [1], then the dependence of ion level $E_{a}$ on the surface-ion distance $z$ can be expressed as below in the atomic unit,

$$
E_{a}=E_{a}(z)=E_{a}(t)=\phi-I+\frac{1}{4\left(z-z_{i m}\right)},
$$

where $\phi, I$ and $z_{i m}$ are work function, ionization potential and the location of image potential (we take $z_{i m}=-2 a_{B}$ and $I=3 \mathrm{eV}$ in calculations hereafter, $a_{B}$ : Bohr radius).

Figure 1 illustrates neutralization rate $\left(=\left\langle n_{a a}(\infty)\right\rangle_{\mathrm{av}}\right)$ as a function of work function $\phi$. From the values of

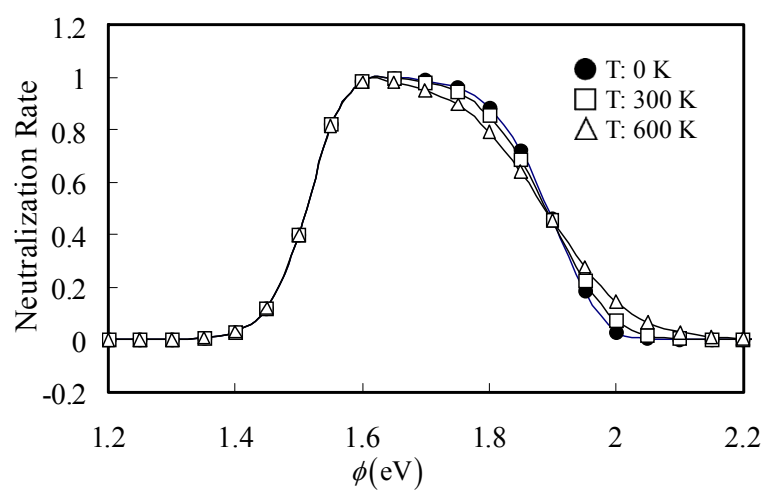

Figure 1. Temperature dependence of neutralization rate $\left(=\left\langle\boldsymbol{n}_{a a}(\infty)\right\rangle_{\mathrm{av}}\right)$ as a function of $\phi$ (= work function) by numerically solving Equations (3.5)-(3.7), where $V_{a k}(t)=$ $V_{a}^{0} \exp \left(-\zeta v^{2} t^{2}\right) \quad\left(V_{a}^{0}=1.2 \mathrm{eV}, \zeta=1 \AA^{-2}\right)$ and ion velocity $v=$ $2 \times 10^{5} \mathrm{~cm} / \mathrm{s}$. $\bigcirc: \mathrm{T}=0 \mathrm{~K}, \square: \mathrm{T}=300 \mathrm{~K}, \triangle: \mathrm{T}=600 \mathrm{~K}$. neutralization rate, it is found that the RCT is numerically confirmed at the range of $1.4 \mathrm{eV} \leq \phi \leq 2 \mathrm{eV}$. As illustrated in Figure 1, neutralization rate is not so sensitive to temperature, but rather slow even in the range of $600 \mathrm{~K}$, which seems consistent with the report by Sulston et al. [17].

Secondly, let us apply our method to the same RCT process when impurity potential is newly introduced. Actually, such a theoretical calculations as evaluating the temperture dependent properties of RCT under the presence of impurity potential have not been reported and discussed. Usually the presence of perturvative Hamiltonian will easily cause the difficulty in the analytical procedures, because the complicated and/or awkward schemes for the evaluation of self energy is usually required. It seems, therefore, to be a very difficult task to obtain exact solution in this case, using conventinal methods such as Green functions based on Keldysh formalism. However, our proposed methods straightforwardly gives exact solution and procedures of analysis are very simple. Let us consider the case where impurity potential exists on crystal surface. Hamiltonian is

$$
\begin{aligned}
\hat{H}= & \hat{H}_{0}+\hat{H}^{\prime}, \\
\hat{H}_{0}= & \sum_{\boldsymbol{k}} E_{\boldsymbol{k}} \hat{C}_{\boldsymbol{k}}^{\dagger} \hat{C}_{\boldsymbol{k}}+E_{a}(z) \hat{C}_{a}^{\dagger} \hat{C}_{a} \\
& +\sum_{\boldsymbol{k}}\left(V_{a \boldsymbol{k}}(z) \hat{C}_{a}^{\dagger} \hat{C}_{\boldsymbol{k}}+V_{\boldsymbol{k} a}(z) \hat{C}_{\boldsymbol{k}}^{\dagger} \hat{C}_{a}\right), \\
\hat{H}^{\prime}= & \sum_{\boldsymbol{k} \boldsymbol{k}^{\prime}} V_{\boldsymbol{k} \boldsymbol{k}^{\prime}} \hat{C}_{\boldsymbol{k}}^{\dagger} \hat{C}_{\boldsymbol{k}^{\prime}} .
\end{aligned}
$$

Concerning $E_{a}(z)$, we take the same expression as Equation (3.9). Based on the above Hamiltonian, we obtain the following equations;

$$
\begin{aligned}
& \frac{\mathrm{d}}{\mathrm{d} t}\left\langle n_{a a}(t)\right\rangle_{\mathrm{av}} \\
& =\frac{\mathrm{i}}{\hbar} \sum_{\boldsymbol{k}} V_{\boldsymbol{k} a}(t)\left\langle n_{\boldsymbol{k} a}(t)\right\rangle_{\mathrm{av}}-\frac{\mathrm{i}}{\hbar} \sum_{\boldsymbol{k}} V_{a \boldsymbol{k}}(t)\left\langle n_{a \boldsymbol{k}}(t)\right\rangle_{\mathrm{av}}, \\
& \frac{\mathrm{d}}{\mathrm{d} t}\left\langle n_{\boldsymbol{k} a}(t)\right\rangle_{\mathrm{av}} \\
& =\frac{\mathrm{i}}{\hbar}\left(E_{\boldsymbol{k}}-E_{a}(z)\right)\left\langle n_{\boldsymbol{k} a}(t)\right\rangle_{\mathrm{av}}+\frac{\mathrm{i}}{\hbar} V_{a \boldsymbol{k}}(t)\left\langle n_{a a}(t)\right\rangle_{\mathrm{av}} \\
& \quad-\frac{\mathrm{i}}{\hbar} \sum_{\boldsymbol{k}^{\prime}} V_{a \boldsymbol{k}^{\prime}}(t)\left\langle n_{\boldsymbol{k} \boldsymbol{k}^{\prime}}(t)\right\rangle_{\mathrm{av}}+\frac{\mathrm{i}}{\hbar} \sum_{\boldsymbol{k}^{\prime}} V_{\boldsymbol{k} \boldsymbol{k}^{\prime}}^{*}\left\langle n_{\boldsymbol{k}^{\prime} a}(t)\right\rangle_{\mathrm{av}} \\
& \frac{\mathrm{d}}{\mathrm{d} t}\left\langle n_{\boldsymbol{k} \boldsymbol{k}^{\prime}}(t)\right\rangle_{\mathrm{av}} \\
& =\frac{\mathrm{i}}{\hbar}\left(E_{\boldsymbol{k}}-E_{\boldsymbol{k}^{\prime}}\right)\left\langle n_{\boldsymbol{k} \boldsymbol{k}^{\prime}}(t)\right\rangle_{\mathrm{av}}+\frac{\mathrm{i}}{\hbar} V_{a \boldsymbol{k}}(t)\left\langle n_{a \boldsymbol{k}^{\prime}}(t)\right\rangle_{\mathrm{av}} \\
& \quad-\frac{\mathrm{i}}{\hbar} V_{\boldsymbol{k}^{\prime} a}(t)\left\langle n_{\boldsymbol{k} a}(t)\right\rangle_{\mathrm{av}}+\frac{\mathrm{i}}{\hbar} \sum_{\boldsymbol{k}^{\prime \prime}} V^{*} \boldsymbol{k}^{\prime \prime}\left\langle n_{\boldsymbol{k}^{\prime \prime} \boldsymbol{k}^{\prime}}(t)\right\rangle_{\mathrm{av}} \\
& \quad-\frac{\mathrm{i}}{\hbar} \sum_{\boldsymbol{k}^{\prime \prime}} V_{\boldsymbol{k}^{\prime} \boldsymbol{k}^{\prime \prime}}\left\langle n_{\boldsymbol{k} \boldsymbol{k}^{\prime \prime}}(t)\right\rangle_{\mathrm{av}},
\end{aligned}
$$


In calculations, we assume $V_{k k^{\prime}}=V_{0}=+10 \mathrm{meV}$ (const and $\left.\operatorname{Im}\left(V_{0}\right)=0\right)$ for shortening calculation time and simplifying the calculation schemes. However, as shown above equations, it is numerically possible for the case of $V_{k k^{\prime}}$ being $\boldsymbol{k}$ and $\boldsymbol{k}^{\prime}$ dependence. Initial conditions are same as stated in the preceding paragraph $(\mathrm{T}=0,300$ and $600 \mathrm{~K}$ ). Additionally, Fermi level $\mu_{0}$ locates at the $E_{\boldsymbol{k}}$ $=0$. Lower and upper limit of band are $-0.29 \mathrm{eV}$ and 0.1 $\mathrm{eV}$, respectively and energy interval $\Delta E$ is $10 \mathrm{meV}$. thus bandwidth $D$ is $0.39 \mathrm{eV}(D=(N-1) \Delta E, N=40)$. Ac- codingly, $-0.29 \mathrm{eV} \leq E_{k} \leq 0.0 \mathrm{eV}($ Fermi Level $)$ is filled with electrons at $T=0 \mathrm{~K}$.

As shown in Equations (3.13)-(3.15), one can easily see that our proposed method is enough simple to evaluate numerical solutions using computer codes and is able to gives exact solutions because impurity potential can be regarded as one body potential.

Figure 2 illustrates neutralization rate $\left(=\left\langle n_{a a}(\infty)\right\rangle_{\mathrm{av}}\right)$ as a function of work function $\phi$ for $V_{0}=+10 \mathrm{meV}$ at $T$ $=0 \mathrm{~K}, 300 \mathrm{~K}$ and $600 \mathrm{~K}$ together with the data of $V_{0}=0$ $\mathrm{meV}$ at $T=0 \mathrm{~K}$. As shown in Figure 2, the shape of neutralization rate drastically changes when impurity potential as small as $10 \mathrm{meV}$ is newly introduced into the system: single peak for $V_{0}=0 \mathrm{meV}$ while about three peaks for $V_{0}=+10 \mathrm{meV}$. From the values of neutralization rate, neutralization is numerically confirmed at the range of about $1.4 \mathrm{eV} \leq \phi \leq 2.4 \mathrm{eV}$ for $V_{0}=+10 \mathrm{meV}$, showing the extension in comparison with the case of $V_{0}=0 \mathrm{meV}$. This extension of neutralization is also numerically confirmed in the previous article [1]; thus it should be remarkable that the presence of impurity potential as small as $10 \mathrm{meV}$ greatly extends the neutralization range. However, neutralization rate is not so sensitive to temperature, which shows same tendency as Figure 1.

In this subsection, for the purpose of verifying the usefulness of our proposed method, we apply this approach to the two cases of RCT: most simplified RCT and RCT with impurity potential newly induced. our proposed method straightforwardly offers exact solutions by such a simple procedure as numerically solving the simultaneous differential equations of (3.5)-(3.7) and (3.13)-(3.15). It should be easily noticed again that the only differences between present work and previous article [1] are initial conditions. Thus we can easily conclude that simultaneous differential equations for seeking temperature dependence of neutralization probability take the same forms as those in previous article [1] except for initial cconditions.

\section{Conclusions}

Starting from Neumann equation, we obtain equation of motion in finite temperature, i.e., Equation (2.5). As illustrated below, it should be obvious that our theoretical

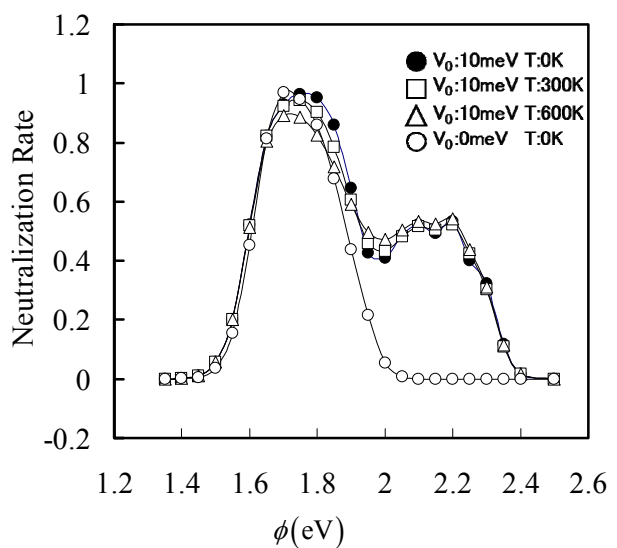

Figure 2. Temperature dependence of neutralization rate $\left(=\left\langle\boldsymbol{n}_{a a}(\infty)\right\rangle_{\mathrm{av}}\right)$ as a function of $\phi \quad(=$ work function) for impurity potential $V_{0}=+10 \mathrm{meV}$ together with the data of $V_{0}=0 \mathrm{meV}$ by numerically solving Equations (3.13)-(3.15), where $V_{a k}(t)=V_{a}^{0} \exp \left(-\zeta v^{2} t^{2}\right) \quad\left(V_{a}^{0}=1.2 \mathrm{eV}, \zeta=1 \AA^{-2}\right)$ and ion velocity $v=2 \times 10^{5} \mathrm{~cm} / \mathrm{s}$. $\bigcirc: T=0 \mathrm{~K} V_{0}=0 \mathrm{meV}$, O: T $=0 \mathrm{~K} V_{0}=+10 \mathrm{meV}, \square: \mathrm{T}=300 \mathrm{~K} V_{0}=+10 \mathrm{meV}, \triangle: \mathrm{T}=$ $600 \mathrm{~K} V_{0}=+10 \mathrm{meV}$.

form takes the same expression as Heisenberg equation of motion except for the $c$-number. Thus our derived equation corresponds to the extension of Heisenberg equation of motion to finite temperature.

$\mathrm{i} \hbar \frac{\partial}{\partial t} \hat{a}_{i}^{\dagger}(t)=\left[\hat{a}_{i}^{\dagger}(t), \hat{H}\right]$ Heisenberg equation of motion $\mathrm{i} \hbar \frac{\mathrm{d}}{\mathrm{d} t}\langle\hat{B}(t)\rangle_{\mathrm{av}}=\langle[\hat{B}, \hat{H}](t)\rangle_{\mathrm{av}}$ Our theoretical form Based on the above theoretical form, we propose a new method to calculate time-dependent properties of $\langle\hat{B}(t)\rangle_{\mathrm{av}}$ in finite temperature by solving numerically simultaneous differential equations straightforwardly derived from Equation (2.5).

Our proposed method consists of numerical procedures to solve these equations without any approximation. Consequently exact solution can be expected, which provide that Hamiltonian is given in an one-electron picture. Being expressed not in the integral manner but in the simultaneous differential equations, our theoretical form can be expected to reduce analytical and/or numerical difficulties greatly even when Hamiltonian has complicated structures or perturbative Hamiltonian is introduced into the system. Furthermore, our proposed method are generally possible to evaluate physical properties in finite temperature even if Hamiltonian includes the terms of phonons and/or photons.

In this work, for the purpose of exhibiting verification and usefulness of our method, we take the two examples: derivation of gap equation in superconductivity and sum rules in electrical conduction. Through these examples, 
our proposed equation seems to be applicable and effective in the various fields.

It should be noted that our proposed method can be easily applied to the many body problem. For example, when there exists the Coulomb repulsive term between $d \uparrow$ and $d \downarrow$ electrons as given as below,

$$
\hat{H}_{\text {coulomb }}=U \hat{C}_{d \uparrow}^{\dagger} \hat{C}_{d \uparrow} \hat{C}_{d \downarrow}^{\dagger} \hat{C}_{d \downarrow},
$$

The terms of $\left\langle n_{d \downarrow d \downarrow d \uparrow \boldsymbol{k} \uparrow}(t)\right\rangle_{\mathrm{av}}$ and $\left\langle n_{d \uparrow d \uparrow d \downarrow k \downarrow}(t)\right\rangle_{\mathrm{av}}$ caused from many body term appear in simultaneous differential equations. In this article, we adopt Hatree Fock approximation

$$
\begin{aligned}
& \left\langle n_{d \downarrow d \downarrow d \uparrow \boldsymbol{k} \uparrow}(t)\right\rangle_{\mathrm{av}} \cong\left\langle n_{d \downarrow d \downarrow}(t)\right\rangle_{\mathrm{av}}\left\langle n_{d \uparrow \boldsymbol{k} \uparrow}(t)\right\rangle_{\mathrm{av}}, \\
& \left\langle n_{d \uparrow d \uparrow d \downarrow \boldsymbol{k} \downarrow}(t)\right\rangle_{\mathrm{av}} \cong\left\langle n_{d \uparrow d \uparrow}(t)\right\rangle_{\mathrm{av}}\left\langle n_{d \downarrow \boldsymbol{k} \downarrow}(t)\right\rangle_{\mathrm{av}} .
\end{aligned}
$$

By introducing such an approximation, we obtain nonlinear simultaneous differential equations with showing closed form, which means that numerical solutions are obtainable. However, the above approximation does not include spin cross term such as $\left\langle n_{d \uparrow d \downarrow}(t)\right\rangle_{\mathrm{av}}$, $\left\langle n_{d \downarrow \boldsymbol{k} \uparrow}(t)\right\rangle_{\text {av }}$ and $\left\langle n_{d \uparrow \boldsymbol{k} \downarrow}(t)\right\rangle_{\mathrm{av}}$. Since more precise approximations than Hartree Fock are considered to have the spin cross terms, we could expect such approximations are expressed as functions of $\left\langle n_{d \downarrow d \downarrow}(t)\right\rangle_{\text {av }}$, $\left\langle n_{d \uparrow \boldsymbol{k} \uparrow}(t)\right\rangle_{\mathrm{av}},\left\langle n_{d \uparrow d \downarrow}(t)\right\rangle_{\mathrm{av}}$ and $\left\langle n_{d \downarrow_{\boldsymbol{k}} \uparrow}(t)\right\rangle_{\mathrm{av}}$ or $\left\langle n_{d \uparrow d \uparrow}(t)\right\rangle_{\mathrm{av}},\left\langle n_{d \downarrow k \downarrow}(t)\right\rangle_{\mathrm{av}},\left\langle n_{d \downarrow d \uparrow}(t)\right\rangle_{\mathrm{av}}$ and $\left\langle n_{d \uparrow \boldsymbol{k} \downarrow}(t)\right\rangle$ in the following manner:

$$
\begin{aligned}
\left\langle n_{d \downarrow d \downarrow d \uparrow \boldsymbol{k} \uparrow}(t)\right\rangle_{\mathrm{av}} \cong & F\left(\left\langle n_{d \downarrow d \downarrow}(t)\right\rangle_{\mathrm{av}},\left\langle n_{d \uparrow \boldsymbol{k} \uparrow}(t)\right\rangle_{\mathrm{av}},\right. \\
& \left.\left\langle n_{d \uparrow d \downarrow}(t)\right\rangle_{\mathrm{av}},\left\langle n_{d \downarrow \boldsymbol{k} \uparrow}(t)\right\rangle_{\mathrm{av}}\right), \\
\left\langle n_{d \uparrow d \uparrow d \downarrow \boldsymbol{k} \downarrow}(t)\right\rangle_{\mathrm{av}} \cong & G\left(\left\langle n_{d \uparrow d \uparrow}(t)\right\rangle_{\mathrm{av}},\left\langle n_{d \downarrow \boldsymbol{k} \downarrow}(t)\right\rangle_{\mathrm{av}},\right. \\
& \left.\left\langle n_{d \downarrow d \uparrow}(t)\right\rangle_{\mathrm{av}},\left\langle n_{d \uparrow \boldsymbol{k} \downarrow}(t)\right\rangle_{\mathrm{av}}\right) .
\end{aligned}
$$

For further research,it seems more importatnt to find and determine optimized function of $F$ and $G$.

Concerning the RCT processes, in which time-dependent physical properties play important roles, we apply two cases: one is the most simplified RCT process and the other are the same process but under impurity potential. In analyzing the first case, no integrations associated with time-dependent terms are found in the obtained simultaneous differential equations, which will greatly reduce numerical instabilities, nuisances and calculation time. Consequently our proposed method seems possible to estimate physical properties even if the system shows complicated time-dependent behaviors. The other case is usually regarded as difficult to analyze be- cause of the presence of impurity potential. The presence of perturbative Hamiltonian such as impurity potential usually causes comlicated and awkward schemes for the evaluation of self energy. However, our propsoed method straightforwardly deduces the solutions without any assumution, which is considered a great merit in analyzing the case where pertubative Hamiltoniam is introduced. Based on our method, the only differences between groud state $(T=0 \mathrm{~K})$ and finite temperature are simple enough to change initial conditions: $\left\langle n_{a a}(-\infty)\right\rangle_{\mathrm{av}}=0$, $\left\langle n_{\boldsymbol{k} a}(-\infty)\right\rangle_{\mathrm{av}}=0$ and $\left\langle n_{\boldsymbol{k} \boldsymbol{k}^{\prime}}(-\infty)\right\rangle_{\mathrm{av}}=\delta_{\boldsymbol{k} \boldsymbol{k}^{\prime}} f_{\mathrm{el}}\left(\beta E_{\boldsymbol{k}}\right)$ at $T \neq 0 \mathrm{~K}$ whereas $\left\langle n_{a a}(-\infty)\right\rangle=0,\left\langle n_{k a}(-\infty)\right\rangle=0$ and $\left\langle n_{k k^{\prime}}(-\infty)\right\rangle=\delta_{k k^{\prime}} \times \theta\left(-E_{k}\right)$ at $T=0 \mathrm{~K}$. Through the analysis of both cases, neutralization process is found to be not so sensitive to temperature up to $600 \mathrm{~K}$ while impurity potential as small as $10 \mathrm{meV}$ greatly changes the neutralization process.

In addition, as already stated in the previous article [1], small energy intervals $\Delta E$ can give accurate and reliable calculation results, which means that the number of electrons $N$ is large because of $N \cong D / \Delta E$ ( $D$ : band width). Furthermore, assuming that the system consists of $N$ electrons, then numerical solutions of $N^{2}$ simultaneous differential equations are required. For example, let us consider actual case. Usually $D$ takes several $\mathrm{eV}$. If we set energy interval $\Delta E=1 \mathrm{meV}$, the value of $N$ is in the range of several thousand, which indicates that we have to solve $10^{6}-10^{7}$ simutaneous differential equations. In this article, we adopt algorithm based on the RungeKutta-Fehlberg formula and solve numerically about $6 \times$ $10^{3}$ simultaneous differential equations, however a fast algorithm to solve a huge number of simultaneous differential equations accurately is strongly desirable from the viewpoints of more precise simulations.

\section{REFERENCES}

[1] S. Kondo and K. Yamada, "Analysis of Resonance Charge Transfer Neutralization on the Basis of Heisenberg Equations of Motion," Progress of Theoretical Physics, Vol. 122, No. 3, 2009, pp. 713-734. doi:10.1143/PTP.122.713

[2] R. Kubo, "Statistical-Mechanical Theory of Irreversible Processes. I. General Theory and Simple Applications to Magnetic and Conduction Problems," Journal of the Physical Society of Japan, Vol. 12, No. 3, 1957, pp. 570586. doi:10.1143/JPSJ.12.570

[3] R. Brako and D. M. Newns, "Charge Exchange in AtomSurface Scattering: Thermal versus Quantum Mechanical Non-Adiabaticity," Surface Science, Vol. 108, No. 2, 1981, pp. 253-270.

[4] R. Brako and D. M. Newns, "Theory of Electronic Processes in Atom Scattering from Surfaces," Reports on Progress in Physics, Vol. 52, No. 6, 1989, pp. 655-698. 


\section{doi:10.1088/0034-4885/52/6/001}

[5] W. Bloss and D. Hone, "Theory of Charge Exchange Scattering from Surfaces," Surface Science, Vol. 72, No. 2, 1978, pp. 277-297.

[6] H. Winter and R. Zimny, Proceedings of XV ICPEAC, Elsevier, Brighton, 1987.

[7] R. Brako, H. Winter and D. M. Newns, "Anisotropic Excitation of Hydrogen $2 p$ after Grazing Ion-Surface Scattering," Europhysics Letters, Vol. 7, No. 3, 1988, pp. 213-218. doi:10.1209/0295-5075/7/3/005

[8] D. M. Newns, "Self-Consistent Model of Hydrogen Chemisorption," Physical Review, Vol. 178, No. 3, 1969, pp. 1123-1135. doi:10.1103/PhysRev.178.1123

[9] L. V. Keldysh, "Diagram Technique for Nonequilibrium," Soviet Physics JETP, Vol. 20, No. 4, 1965, pp. 10181026.

[10] T. Sakai, M. Sakaue, H. Kasai and A. Okiji, "Theory of Dynamics of Electron Wave Packets in Time-Resolved Two-Photon Photoemission via Image States," Applied Surface Science, Vol. 169-170, 2001, pp. 57-62. doi:10.1016/S0169-4332(00)00633-4

[11] E. A. García, C. G. Pascual, P. G. Bolcatto, M. C. G. Passeggi and E. C. Goldberg, "Ion Fractions in the Scattering of Hydrogen on Different Reconstructed Silicon Surfaces," Surface Science, Vol. 600, No. 10, 2006, pp. 2195-2206. doi:10.1016/j.susc.2006.03.008
[12] M. Krawiec, M. Jalochowski and M. Kisiel, "High Resolution Scanning Tunneling Spectroscopy of Ultrathin $\mathrm{Pb}$ on $\operatorname{Si}(111)-(6 \times 6)$ Substrate," Surface Science, Vol. 600, No. 8, 2006, pp. 1641-1645.

[13] T. Mii and K. Makoshi, "An Interpolation Formula for Electron Transfer in Atom-Surface Collision and Its Validity," Surface Science, Vol. 363, No. 1-3, 1996, pp. 145 149.

[14] E. A. García, M. A. Romero, C. González and E. C. Goldberg, "Neutralization of $\mathrm{Li}^{+}$Ions Scattered by the $\mathrm{Cu}(100)$ and (111) Surfaces: A Localized Picture of the Atom-Surface Interaction," Surface Science, Vol. 603, No. 4, 2009, pp. 97-605. doi:10.1016/j.susc.2008.12.022

[15] A. Sindona and G. Falcone, "Evidences of a Double Resonant Ionization Mechanism in Sputtering of Metals," Surface Science, Vol. 529, No. 3, 2003, pp. 471-489. doi:10.1016/S0039-6028(03)00334-0

[16] A. Sindona, P. Riccardi, S. Maletta and G. Falcone, "Double Resonant Neutralization in Hyperthermal Energy Alkali Ion Scattering at Clean Metal Surfaces," Nuclear Instruments and Method in Physics Research B, Vol. 267, No. 4, 2009, pp. 578-583. doi:10.1016/j.nimb.2008.11.053

[17] K. W. Sulston and F. O. Goodman, "Electronic Bath approach to Thermal Effects in Ion-Surface Scattering," The Journal of Chemical Physics, Vol. 112, No. 5, 2000, p. 2486. 